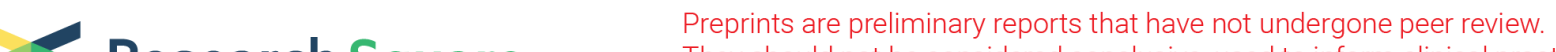 Research Square
or referenced by the media as validated information.
}

\section{A Dual-Fluorescence Labeling Pseudovirus for Real- Time Imaging of Single SARS-CoV-2 Entry in Respiratory Epithelial Cells}

\section{Yingxin Ma}

Beijing University of Chemical Technology

\section{Guobin Mao}

Shenzhen Institutes of Advanced Technology, Chinese Academy of Sciences

\section{Guoqiang Wu}

Shenzhen Institutes of Advanced Technology, Chinese Academy of Sciences

\section{Minghai Chen}

Shenzhen Institute of Synthetic Biology, Shenzhen Institutes of Advanced Technology, Chinese

Academy of Sciences

\section{Fujun Qin}

Shenzhen Institutes of Advanced Technology, Chinese Academy of Sciences

\section{Xian-En Zhang ( $\nabla$ zhangxe@ibp.ac.cn )}

National Laboratory of Biomacromolecules, CAS Center for Excellence in Biomacromolecules, Institute of Biophysics, Chinese Academy of Sciences, Beijing, 100101

\section{Article}

Keywords: Pseudovirus Strategy, Lipophilic Dye, mCherry, Viral Entry, Endocytosis

Posted Date: December 7th, 2020

DOl: https://doi.org/10.21203/rs.3.rs-112689/v1

License: (a) (1) This work is licensed under a Creative Commons Attribution 4.0 International License. Read Full License 


\section{Abstract}

The pseudovirus strategy makes studies of highly pathogenic viruses feasible without the restriction of high-level biosafety facility, thus greatly contributing to virology and being used in research of SARS-CoV2. Here, we generated a dual-color pseudo-SARS-CoV-2 virus using an HIV-1 pseudovirus production system and the SARS-CoV-2 spike (S) glycoprotein, of which the membrane was labeled with lipophilic dye (DiO) and the genomic RNA-related viral protein R (Vpr) of the viral core were fused with mCherry. With this dual-color labeling strategy, not only the movement of the whole virus but also the fate of the labeled components can be traced. The pseudovirions were applied to track viral entry at a single particle level in four types of the human respiratory cells: nasal epithelial cells (HNEpC), pulmonary alveolar epithelial cells (HPAEpiC), bronchial epithelial cells (BEP-2D), and oral epithelial cells (HOEC). PseudoSARS-CoV-2 entered into the host cell and released viral core into the cytoplasm $₫$ which clearly indicates that the host entry mainly occurred through endocytosis. The infection efficiency was found to be correlated with the expression of the known receptor of SARS-CoV-2, angiotensin-converting 2 (ACE2) on the host cell surface. We believe that the dual-color fluorescence labeled pseudovirus system created in this study can be a useful tool in SARS-CoV-2/COVID-19 for many purposes.

\section{Introduction}

Since 2020, COVID-19 has raged globally, posing a huge threat to human health and severely damaging the world economy. ${ }^{1-3}$ Understanding the infection mechanism has become one of top priorities. While determining that the pathogen was a new coronavirus, later named SARS-CoV-2, scientists immediately confirmed that SARS-CoV-2 uses the same cell entry receptor-angiotensin converting enzyme II (ACE2)-as SARS-CoVby its spike (S) protein. ${ }^{4-6}$ SARS-CoV-2 enters host cells mainly through endocytosis after binding to the ACE2 receptor, and the proposed model is based on lysosomotropic inhibitor treatment of infected cells. ${ }^{7}$ However, how does the virus enter the cell after binding to the receptor has never been visualized through live imaging. In addition, how sensitive are different parts of the respiratory tract to the virus remains undocumented. Our interest is to conduct systematic investigations of these issues.

Due to the high pathogenicity of SARS-CoV-2, it needs to be operated in biosafety level 3 laboratory (BSL$3)$, which restricts many routine researches. ${ }^{8-11}$ Pseudovirus strategy can provide a safe manner to study highly pathogenic viruses without high-level biosafety facility. They usually refer to a retrovirus that can integrate the envelope glycoprotein of another virus to form an envelope from foreign virus, and the genome retains the characteristics of the retrovirus itself. ${ }^{12-14}$ Pseudovirus has part of the functional structure of the target virus, but have lost the function of reproducing in host cells, so they can be used to identify the function of virus components. ${ }^{15,16}$ SARS-CoV-2 pseudovirus has been successfully applied to determine the receptor and infection pathway, evaluate neutralizing antibodies, and construct recombinant vaccine. ${ }^{7,17,18}$ In our previously studies, we have developed multi-color labeling methods for viruses to visualize key molecular processes of viral infection and to elucidate the basic virological issues. ${ }^{19-23}$ 
In this study, a dual-color pseudo-SARS-CoV-2 was generated by incorporating SARS-CoV-2 S protein into HIV-1 pseudovirus. Viral lipid envelope and the Vpr protein were labeled with a lipophilic membrane dye (DiO) and mCherry fluorescence protein, respectively. The dual-labeled virus was used to infect the human airway epithelium cultures to image the entry pathway and infection efficiency at a single particle level in four different respiratory epithelial cells, including human nasal epithelial cells (HNEpC), human oral epithelial cells (HOEC), human bronchial epithelial cells (BEP-2D), and human pulmonary alveolar epithelial cells (HPAEpiC). The entry pathway and cell-type susceptibility of SARS-CoV-2 to respiratory tract cells were first identified and systematically investigated by single virus tracking.

\section{Results}

Construction and characterization of pseudo-SARS-CoV-2 virus. The SARS-CoV-2 S plasmid, encoding the $S$ protein, was synthesized and transfected into the $293 \mathrm{~T}$ cells. Western blot was used to analyze the expression of SARS-CoV-2 S protein. The major band, reflecting the full-length S protein (180 kDa), was detected using the rabbit anti-S antibody. $S$ proteins were incorporated into the pseudovirions while assembling the virus, and the efficiency was evaluated using a monoclonal mouse anti-S1 antibody. The full-length $S$ protein was incorporated into the pseudotyped virus; however, the majority of $S$ proteins in pseudovirions were cleaved (Fig. 1A). Pseudovirions were further characterized by negative staining in TEM. The round-shaped virus particles had an average size of $100 \mathrm{~nm}$, and the cobbled surface structure of SARS-CoV-2 S proteins had an average size of $15 \mathrm{~nm}$ (Fig. 1B).

Pseudo-SARS-CoV-2 were overlaid onto coverslips and stained for immunofluorescence (IF) with an anti$S$ antibody. The lipid envelope of the pseudotyped virus was labeled using DiO and the majority of DiO co-localized with the S protein (Fig. 1D). Pseudovirions were also stained with an anti-p24 antibody and $\mathrm{DiO}$, and we found that they were well co-localized. Then, pseudo-SARS-CoV-2 were exposed to combined anti-S and anti-p24 immunofluorescence staining, and S protein was found co-localized with p24 protein (Supplementary Fig. 1). These results demonstrated that SARS-CoV-2 S proteins were successfully incorporated into the pseudovirions.

Dual-labeled fluorescent infectious pseudovirions. We generated dual-labeled fluorescent infectious pseudovirions to visualize the dynamic entry of the SARS-CoV-2 virus into the host cells. The Vpr protein of the viral core was labeled by fusing with the mCherry fluorescence protein, and the Vpr-mCherry complex was encapsulated into the pseudo-SARS-CoV-2 during virus assembly. Immunofluorescence staining with an anti-S antibody or anti-p24 antibody verified the successful labeling of the Vpr proteins with mCherry fluorescence protein (Fig. 1D and Supplementary Fig. 1). The second color was obtained by labeling the lipid envelope with $\mathrm{DiO}$, and fluorescence co-localization of Vpr-mCherry and DiO verified the successful construction of dual-fluorescent pseudo-SARS-CoV-2 (Fig. 1D).

Pseudo-SARS-CoV-2 with/without fluorescence were analyzed using RT-PCR to determine whether fluorescent labeling affected the viral infectivity. The results showed that the titer activity of single-labeled 
particles (Vpr-mCherry or DiO) and dual-labeled particles were similar to that of unlabeled pseudovirions, demonstrating that the labeling did not impair virus transmission (Fig. 1C).

\section{Real-time imaging of pseudo-SARS-CoV-2 entry into upper respiratory cells, human nasal epithelial cells} (HNEpC). Pseudo-SARS-CoV-2 was used to infect different respiratory epithelial cells to conduct real-time imaging of the viral entry process. The dynamic entry of dual-labeled pseudo-SARS-CoV-2 was first tracked in the upper respiratory cells, human nasal epithelial cells (HNEpC). Only the co-localized signals of Vpr-mCherry and DiO were considered as single virus. A virus particle was observed on the surface of the HNEpC cell membrane, exhibiting the entry of pseudo-SARS-CoV-2 into HNEpC cells. Fig. 2A-2B and Supplementary Movie 1 show the trajectory of pseudovirions. The virus particle was first attached to the HNEpC cell membrane and was rapidly transported into the cytoplasm (Fig. 2C). The results of mean square displacement (MSD) indicated that the pseudovirions were endocytosed into HNEpC cells through active transport (Fig. 2D).

A common virus, vesicular stomatitis virus glycoprotein (VSV-G) pseudovirions, was used as the control virus. The control virus was also labeled with DiO and Vpr-mCherry and used to infect HNEpC cells. We observed similar endocytic patterns in HNEpC cells (Supplementary Fig. 1A-1D and Supplementary Movie 9). The results suggested that pseudo-SARS-CoV-2 entered HNEpC cells through endocytosis.

\section{The release of viral core from the envelope during endocytic entry revealed by single particle tracking.}

During the endocytic entry of pseudovirions, we visualized the release of viral core from the envelope into the cytoplasm in various respiratory epithelial cells. The release was first observed in HNEpC cells by temporal tracking of dual-labeled pseudo-SARS-CoV-2. Virus particles with co-localized signals of VprmCherry and DiO were imaged in the cytoplasm of HNEpC cells. During virus transportation, Vpr-mCherry (red) was separated from DiO (green), indicating the release of the viral core from the envelope (Fig. 2E-2F and Supplementary Movie 2). Fig. 2G-2I show the dynamic trajectories, velocities, and MSD of VprmCherry and the envelope with DiO. Both fluorescent dots had different trajectories, velocities, and MSD after their separation. The results suggested that the viral core successfully escaped from the endosomes and was released into the cytoplasm of HNEpC cells, and the dynamic process was necessary for productive infection.

The release process was further confirmed by using VSV-G pseudovirions as the control virus to infect HNEpC cells. While tracking the dual-labeled control virus, Vpr-mCherry (red) separated from DiO (green) in the host cells (Supplementary Fig. 1E-1I and Movie S10). These results suggested that the viral core of pseudo-SARS-CoV-2 escaped from the envelope and were released into the cytoplasm of HNEpC cells. 
The entry process of pseudo-SARS-CoV-2 into lower respiratory cells, human pulmonary alveolar epithelial cells (HPAEpiC). The entry process of dual-labeled pseudo-SARS-CoV-2 was tracked in HPAEpiC cells, and the dynamic behavior of the virus was visualized through real-time imaging in the lower respiratory cells. Fig. 3A-3B and Supplementary Movie 3 show the trajectories of virus particles in HPAEpiC cells. Initially, the virus particles attached to the cell membrane and then were rapidly transported into the cytosol (Fig. $3 \mathrm{C}$ ). The MSD results suggested that the pseudovirions were endocytosed into the host cells through active transport (Fig. 3D).

Then, the release process of pseudo-SARS-CoV-2 was imaged in human HPAEpiC cells. We visualized the separation of the Vpr-mCherry signal (red) from the DiO signal (green) during virus transportation in the cytoplasm, indicating that the viral core was released from the envelope (Fig. 3E-3F and Supplementary Movie 4). The different dynamic trajectories, velocities, and MSD for these two parts during the separation behavior in these cells are shown in Fig. 3G-3I, demonstrating the dynamic release of the viral core into the cytoplasm.

The control virus, DiO/Vpr-mCherry dual-labeled VSV-G pseudovirions were used to infect HPAEpiC cells and showed similar entry and release processes (Supplementary Fig. 3 and Supplementary Movie 11-12). The results suggested that pseudo-SARS-CoV-2 entered HPAEpiC cells through endocytosis.

Single-particle tracking of pseudo-SARS-CoV-2 in the other two respiratory epithelial cells. The duallabeled pseudo-SARS-CoV-2 was also used to infect human bronchial epithelial cells (BEP-2D), a type of lower respiratory cells, and human oral epithelial cells (HOEC), a type of upper respiratory cells. The endocytic patterns of pseudo-SARS-CoV-2 in these two cell lines were visualized through single-particle tracking. Virus particles were attached to the cell membrane, rapidly transported into the cytosol, followed by the sequential release of the viral core from the envelope (Fig. 4-5 and Supplementary Movie 5-8). The control group captured similar phenomena through real-time imaging of dual-labeled VSV-G pseudotyped virus in both BEP-2D cells and HOECs (Supplementary Fig. 4-5 and Supplementary Movie 13-16). The results suggested that pseudo-SARS-CoV-2 entered BEP-2D cells and HOEC cells through endocytosis. The endocytic entry of pseudo-SARS-CoV-2 in different types of respiratory epithelial cells was visualized and it was found to exhibit similar sequential patterns.

The receptor ACE2 was critical for the efficiency of SARS-CoV-2 productive infection. We performed a high-throughput analysis of dual-labeled pseudo-SARS-CoV-2 in different respiratory epithelial cells to study the efficiency of viral productive infection. The infected cells were fixed at different time points, and viral entry efficiency was analyzed in HNEpC, HPAEpiC, BEP-2D, and HOEC, respectively. At each time point, 500 host cells were randomly selected for statistical analysis, and the results were collected during 0-180 min post-infection window. In the cytoplasm, the obvious increase in the co-localized signals of DiO and Vpr-mCherry at 0-60 min was due to the entry of virus particles into the host cells. Also, there was a 
significant decrease in the co-localized dots at 60 - $90 \mathrm{~min}$, indicating that the viral core was released from the envelope during this period. The productive infection of pseudo-SARS-CoV-2 showed a noticeable difference in HNEpC, HPAEpiC, BEP-2D cells, and HOEC (Fig. 6A). However, similar levels in productive infection were observed among the four cells types infected with VSV-G pseudotyped virus (control) (Fig. 6B). The results suggested that the differences between pseudo-SARS-CoV-2 and VSV-G pseudovirions were probably mediated by the interaction between S proteins and the SARS-CoV-2 receptors on these respiratory epithelial cells.

ACE2, the receptor for SARS-CoV-2, in these respiratory epithelial cells was tested using western blot. In Fig. 6C, the expression of the ACE2 receptor on these cells was consistent with SARS-CoV-2 productive infection based on statistical analysis. Next, pseudo-SARS-CoV-2 with equal numbers of RNA copies were used to infect respiratory epithelial cells to further determine the efficiency of virus entry (Fig. 6D). At $2 \mathrm{~h}$ post-infection, total RNA was extracted from the host cells, and intracellular viral RNA copy number was analyzed by RT-PCR. The intracellular viral RNA copy number of pseudo-SARS-CoV-2 in each host was positively correlated with the expression of the ACE2 receptor, indicating that the ACE2 receptor was critical for the efficiency of SARS-CoV-2 productive infection.

\section{Discussion}

In this work, we constructed dual-color pseudo-SARS-CoV-2 to investigate the entry pathway of the virus as well as the infection efficiency in respiratory epithelial cells under the conditions of BSL-2. Western blot and TEM results showed SARS-CoV-2 S protein had been incorporated into the pseudovirions and form the cobbled surface structure in the HIV-1 pseudovirus system. Additionally, the lipid envelope staining or immunofluorescence staining was performed to verify the fluorescence labeling of virus particles. Results suggested that the constructed pseudo-SARS-CoV-2 possessed high fluorescence co-localization efficiency. These particles also owned high infectivity, due to the labeling strategies exerted no significant interference to viral structure and function. Thus, dual-fluorescent pseudo-SARS-CoV-2 provides a suitable tool for imaging the dynamic process of virus entry and capture details at the single-particle level.

As an air-transmitted virus, SARS-CoV-2 infection must happen through interactions between the virus and respiratory cells. Whether it only infects particular types of respiratory cells remains elusive. By observing patterns of virus entry among four types of respiratory cells (HNEpC, HOEC, BEP-2D cells and HPAEpiC), we found that pseudo-SARS-CoV-2 exhibit identical sequential process, suggesting there is no or little preference among these cells. Meanwhile, we showed that SARS-CoV-2 productive infection in different types of respiratory epithelial cells occurred through endocytosis. This result is consistent with report from Ou et al who showed that drugs blocking endocytosis can prevent entry of SARS-CoV-2. ${ }^{7}$

Statistical analysis and RT-PCR results showed that there was a significant difference in the infection efficiency of various respiratory epithelial cells, and pseudo-SARS-CoV-2 preferentially infected HNEpC. This could be attributed to the different levels of expression of SARS-CoV-2 receptors on these cells, because pseudo-SARS-CoV-2 infection required interaction between S protein and ACE2. In contrast, VSV- 
G pseudotyped virus failed to differentiate cells with different amount of ACE2 receptors. The results were consistent with early reports that the nose contained the highest percentage of ACE2-expression cells in the airways, and the nasal surfaces were the dominant initial site for SARS-CoV-2 respiratory tract infection. ${ }^{2,16,24}$

Our study provides an excellent tool to systematically visualize the progression of SARS-CoV-2 infection in live cells. In the future, other viral components could be included in the pseudovirions system to investigate additional processes of the viral life cycle at the single-particle level. On the other hand, although we found endocytosis via interaction between SARS-CoV-2 S protein and ACEs is the major driving force of SARS-CoV-2 infection, we have not evaluated effects of endocytosis inhibitors, neutralizing antibody, or mutations of $S$ protein during virus entry into cells. These relevant studies should be performed using live SARS-CoV-2 virus under the conditions of BSL-3.

In conclusion, we constructed a dual-color fluorescence labelled pseudo-SARS-CoV-2 to study virus entry and cell susceptibility under BSL-2 conditions. Dynamic process of virus entry was visualized, imaged, and analyzed in live cells at single virion level. We observed similar patterns of entry pathways in different respiratory epithelial cells through endocytosis. There was a positive correlation between infection efficiency of the SARS-CoV-2 and expression level of the ACE2 receptor. The method may be further developed to elucidate the life cycle of SARS-CoV-2 at a single particle level.

\section{Methods}

Plasmids. The pcDNA3.1(+)-Vpr-mCherry was produced to express the Vpr-mCherry protein. pNL4-3-KFS $(\triangle$ env), pSARS-CoV-2-S, and pVSV-G were used to assemble the pseudovirions.

Cell culture. Immortalized human bronchial epithelial cells (BEP-2D) and human alveolar epithelial cells (HPAEpiC) were obtained from BioDee (Beijing, China). Human oral epithelial cells (HOEC) were obtained from BLUEFBIO (Shanghai, China). Human nasal epithelial cell line (HNEpC) was obtained from Tongpai Biotechnology (Shanghai, China). All cells were cultured in DMEM supplemented with 10\% FBS (Gibco) at $37^{\circ} \mathrm{C}$ in a $5 \% \mathrm{CO}_{2}$ humidified atmosphere.

Pseudovirions preparation. Virus-related experiments were conducted in the BSL-2 laboratories. The 293T cells $\left(5 \times 10^{6}\right)$ were co-transfected with $10 \mu \mathrm{g}$ of pNL4-3-KFS ( $\triangle$ env) and $10 \mu \mathrm{g}$ of pSARS-CoV-2-S using lipofectamine 3000 (Invitrogen) to construct pseudo-SARS-CoV-2. Also, 293T cells were co-transfected with $10 \mu \mathrm{g}$ of pNL4-3-KFS ( $\triangle \mathrm{env}$ ) and $10 \mu \mathrm{g}$ of pVSV-G to generate VSV-G pseudovirions. Virus particles were harvested at $24 \mathrm{~h}$ post-transfection. 
Fluorescent labeling and purification. Cells were transfected with $10 \mu \mathrm{g}$ of pNL4-3-KFS ( $\triangle \mathrm{env}), 10 \mu \mathrm{g}$ of pSARS-CoV-2-S, and $2 \mu \mathrm{g}$ of pcDNA3.1(+)-Vpr-mCherry to produce pseudo-SARS-CoV-2 (Vpr-mCherry). Next, $10 \mathrm{~mL}$ of virus particles were stained with $0.5 \mu \mathrm{L}$ of DiO (Invitrogen) for $1 \mathrm{~h}$ at $37^{\circ} \mathrm{C}$ to label the lipid envelope. Equal volumes of PEG 20000 solution (PEG $20000(20 \%(\mathrm{w} / \mathrm{v}))$ were dissolved in $0.9 \% \mathrm{NaCl}$ solution) and the virus was mixed at $4{ }^{\circ} \mathrm{C}$ overnight, and the solution was centrifuged at $9000 \mathrm{rpm}$ for 20 min. The purified virus was resuspended in $1 \mathrm{~mL}$ of Opti-MEM. Cells were transfected with $20 \mu \mathrm{g}$ of pNL43-KFS ( $\triangle$ env), $10 \mu \mathrm{g}$ of pVSV-G, and $2 \mu \mathrm{g}$ of pcDNA3.1(+)-Vpr-mCherry to prepare VSV-G pseudovirions (Vpr-mCherry). DiO was also used to prepare VSV-G pseudovirions (DiO).

Immunofluorescence staining. Pseudo-SARS-CoV-2 were fixed in $4 \%$ paraformaldehyde, permeabilized using $0.1 \%$ Triton-X100, and blocked with FBS containing 10\% BSA. Next, they were incubated overnight with primary antibody at $4^{\circ} \mathrm{C}$, followed by incubation with a secondary antibody for $2 \mathrm{~h}$ at $37^{\circ} \mathrm{C}$. PseudoSARS-CoV-2 were stained using rabbit anti-SARS-CoV-2 $\mathrm{S}$ antibody (1:200, Sinobio) and/or mouse antip24 antibody (1:200, Abcam). Goat anti-mouse IgG conjugated with Alexa Fluor 488 (1:500, CST), rabbit anti-mouse IgG conjugated with Alexa Fluor $488(1: 500, C S T)$, goat anti-rabbit IgG conjugated with Alexa Fluor 488 (1:500, CST), and goat anti-rabbit IgG conjugated with Alexa Fluor 555 (1:500, CST) were used as secondary antibodies.

Virus infection and fluorescence imaging. Respiratory epithelial cells were plated into a confocal dish and incubated with pseudo-SARS-CoV-2 (DiO-mCherry) for 30 min at $4^{\circ} \mathrm{C}$. Unbound particles were removed and replaced with fresh media before incubation at $37^{\circ} \mathrm{C}$ in a $5 \% \mathrm{CO}_{2}$ atmosphere. Infected cells were imaged under an UltraView Vox spinning disk confocal laser scanning system using a Nikon Ti-e microscope with $60 \times$ objective. DiO and mCherry were excited at $488 \mathrm{~nm}$ and $561 \mathrm{~nm}$, respectively. Realtime imaging was usually performed at an interval of $10 \mathrm{~s}$ for $15 \mathrm{~min}$ to minimize photobleaching.

Statistical analysis. Respiratory epithelial cells were incubated with dual-labeled fluorescent pseudoSARS-CoV-2 (DiO-mCherry) for $30 \mathrm{~min}$ at $4^{\circ} \mathrm{C}$ and removed and incubated at $37^{\circ} \mathrm{C}$ for different time intervals $(0,30,60,90,120$, and $180 \mathrm{~min})$. Finally, the cells were fixed in $4 \%$ formaldehyde for imaging.

Western blot. The samples were boiled for $10 \mathrm{~min}$, separated using 10\% SDS-PAGE gel, and transferred to a PVDF membrane. After blocking the non-specific sites with 5\% (w/v) skim milk, the membranes were incubated with the primary antibody, followed by HRP-conjugated secondary antibody. The enhanced chemiluminescence detection kit (Beyotime Biotechnology) was used to detect the bands, which were visualized using a ChemStudio Imaging System (Analytik Jena AG). Rabbit anti-SARS-CoV-2 S antibody 
(1:1000; ABclone), mouse anti-SARS-CoV-2 S1 antibody (1:1000; Sinobio), rabbit anti-ACE2 antibody (1:1000; Abcam), mouse anti-GAPDH antibody (1:5000; TransGen Biotech), mouse anti- $\beta$-actin antibody (1:2000; CST), and mouse anti-p24 antibody (1:2000; Abcam) were used as primary antibodies. HRPlinked horse anti-mouse IgG and HRP-linked horse anti-rabbit IgG (1:2000; CST) were used as the secondary antibodies.

Transmission electron microscopy analysis. Pseudo-SARS-CoV-2 was concentrated using the PEG 20000 solution, followed by ultrafiltration using an Amicon Ultra-4 centrifugal filter device (50 kDa, Millipore). The carbon support copper grid was placed on the purified pseudovirions and incubated for $10 \mathrm{~min}$. Then, the pseudovirions loaded on the carbon support copper grid were negatively stained with $2 \%$ phosphotungstate (PTA) for $30 \mathrm{~s}$. The samples were observed on a $200 \mathrm{kV}$ JEM-F200 transmission electron microscope.

Real-time PCR. Cellular total RNA of the infected cells was extracted $24 \mathrm{~h}$ post-transfection, and quantitative real-time PCR analysis was performed using a HiScript II One-Step qRT-PCR Probe Kit (Vazyme Biotech). To confirm the virus entry, cellular RNA was extracted $2 \mathrm{~h}$ post-transfection and analyzed using RT-PCR. The DNA sequences used were as follows: The primer HIV sense (TTT GAC TAG CGG AGG CTA GAA G), HIV antisense (CCC TGG CCT TAA CCG AAT TTT), and a specific TaqMan probe (FAM-CGC TTA ATA CCG ACG CTC TCG C-TAMRA).

\section{References}

1. Zou, L.R. et al. SARS-CoV-2 Viral Load in Upper Respiratory Specimens of Infected Patients. New Engl J Med 382, 1177-1179 (2020).

2. Wu, F. et al. A new coronavirus associated with human respiratory disease in China (vol 579, pg 265, 2020). Nature 580, E7-E7 (2020).

3. Lu, R.J. et al. Genomic characterisation and epidemiology of 2019 novel coronavirus: implications for virus origins and receptor binding. Lancet 395, 565-574 (2020).

4. Zhao, Y. et al. Single-Cell RNA Expression Profiling of ACE2, the Receptor of SARS-CoV-2. Am J Resp Crit Care 202, 756-759 (2020).

5. Zhou, P. et al. A pneumonia outbreak associated with a new coronavirus of probable bat origin. Nature 579, 270-+ (2020).

6. Lukassen, S. et al. SARS-CoV-2 receptor ACE2 and TMPRSS2 are primarily expressed in bronchial transient secretory cells. Embo J 39 (2020).

7. Ou, X.Y. et al. Characterization of spike glycoprotein of SARS-CoV-2 on virus entry and its immune cross-reactivity with SARS-CoV. Nat Commun 11 (2020). 
8. Thao, T.T.N. et al. Rapid reconstruction of SARS-CoV-2 using a synthetic genomics platform. Nature (2020).

9. Rockx, B. et al. Comparative pathogenesis of COVID-19, MERS, and SARS in a nonhuman primate model. Science 368, 1012-+ (2020).

10. Singh, M., Bansal, V. \& Feschotte, C. A Single-Cell RNA Expression Map of Human Coronavirus Entry Factors. Cell Rep 32 (2020).

11. Jaume, M. et al. Anti-Severe Acute Respiratory Syndrome Coronavirus Spike Antibodies Trigger Infection of Human Immune Cells via a pH-and Cysteine Protease-Independent Fc gamma R Pathway. J Viro/ 85, 10582-10597 (2011).

12. Menachery, V.D. et al. MERS-CoV Accessory ORFs Play Key Role for Infection and Pathogenesis. Mbio 8 (2017).

13. Nie, Y.C. et al. Highly infectious SARS-CoV pseudotyped virus reveals the cell tropism and its correlation with receptor expression. Biochem Bioph Res Co 321, 994-1000 (2004).

14. Siu, Y.L. et al. The M, E, and N Structural Proteins of the Severe Acute Respiratory Syndrome Coronavirus Are Required for Efficient Assembly, Trafficking, and Release of Virus-Like Particles. $J$ Virol 82, 11318-11330 (2008).

15. Wang, C. et al. MERS-CoV virus-like particles produced in insect cells induce specific humoural and cellular imminity in rhesus macaques. Oncotarget 8, 12686-12694 (2017).

16. Richard, M. et al. Influenza A viruses are transmitted via the air from the nasal respiratory epithelium of ferrets. Nat Commun 11 (2020).

17. Nie, J.H. et al. Establishment and validation of a pseudovirus neutralization assay for SARS-CoV-2. Emerg Microbes Infec 9, 680-686 (2020).

18. Zhu, F.C. et al. Safety, tolerability, and immunogenicity of a recombinant adenovirus type- 5 vectored COVID-19 vaccine: a dose-escalation, open-label, non-randomised, first-in-human trial. Lancet 395 , 1845-1854 (2020).

19. Ma, Y. et al. Real-Time Imaging of Single HIV-1 Disassembly with Multicolor Viral Particles. ACS Nano $10,6273-6282(2016)$.

20. Ma, Y. et al. Quantum Dot Nanobeacons for Single RNA Labeling and Imaging. J Am Chem Soc 141, 13454-13458 (2019).

21. Ma, Y. et al. Live cell imaging of single genomic loci with quantum dot-labeled TALEs. Nat Commun 8, 15318 (2017).

22. Yin, W. et al. Real-time imaging of individual virion-triggered cortical actin dynamics for human immunodeficiency virus entry into resting CD4 T cells. Nanoscale 12, 115-129 (2020).

23. Li, X. et al. HIV-1 viral cores enter the nucleus collectively through the nuclear endocytosis-like pathway. Sci China Life Sci (2020).

24. Sungnak, W. et al. SARS-CoV-2 entry factors are highly expressed in nasal epithelial cells together with innate immune genes. Nat Med 26, 681-+ (2020). 


\section{Declarations}

\section{Acknowledgements}

This work was supported by National Key Research and Development Program of China (2017YFA0205503), National Natural Science Foundation (21890743, 21705110), Strategic Priority Research Program of the Chinese Academy of Sciences, China (XDB29050100), Natural Science Foundation of Guangdong (2018B030306046), China Postdoctoral Science Foundation (2019M663154).

\section{Author contributions}

Y. X. M. and X. E. Z. conceived and designed the experiments. G. B. M. and G. Q. W. performed the experiments. G. B. M., G. Q. W., M. H. C., and F. J. Q. analyzed the data. Y. X. M. and X. E. Z. wrote the paper.

\section{Additional information}

Competing financial interests: The authors declare no competing financial interests.

\section{Figures}




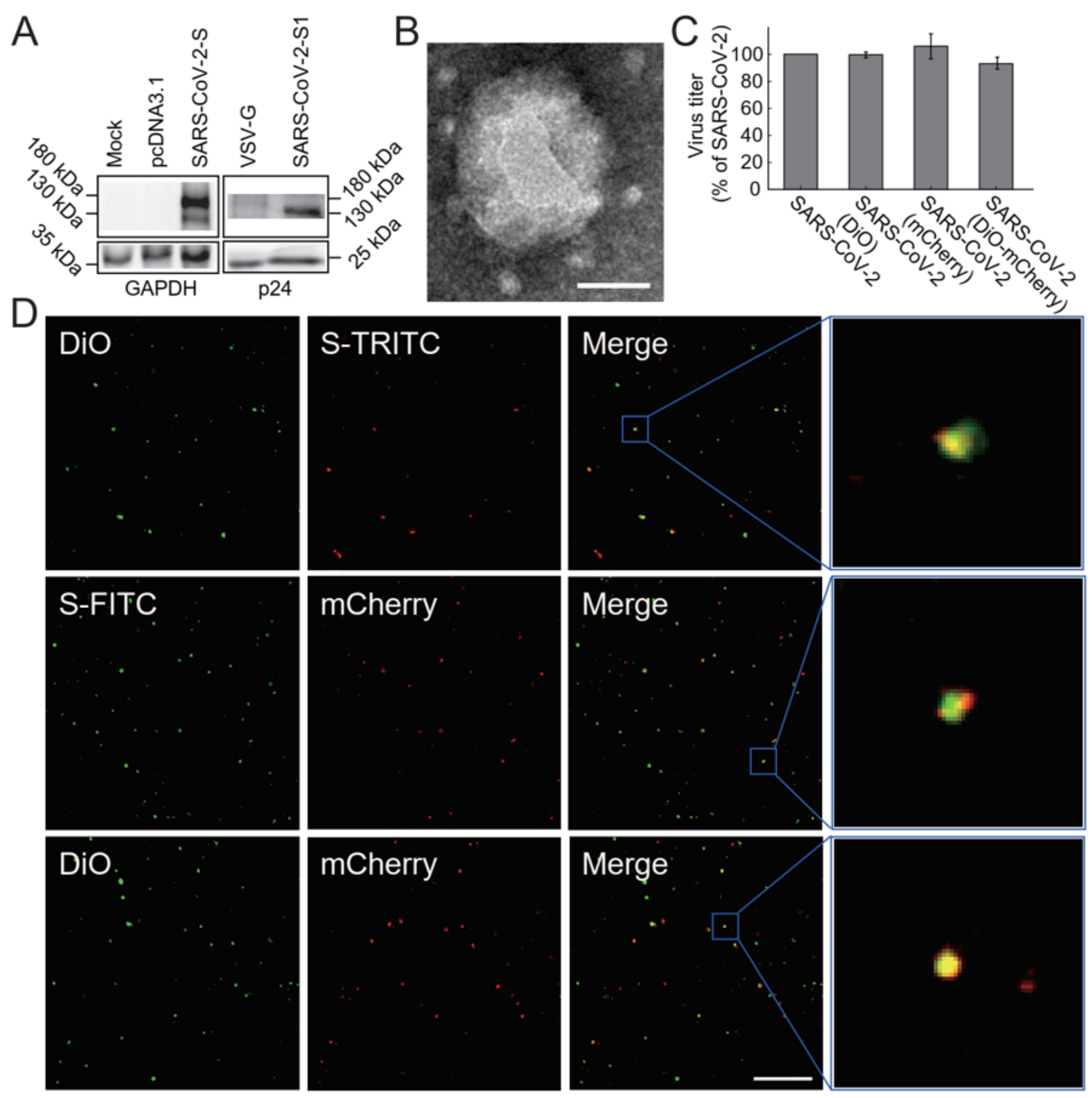

Figure 1

(A) Western Blot analysis of the SARS-CoV-2 S protein in the cell lysates (left). Western Blot analysis of the SARS-CoV-2 S1 protein in pseudovirions (right). (B) TEM images of the negatively stained pseudoSARS-CoV-2 (Scale bar: $50 \mathrm{~nm}$ ). (C) Titers of pseudo-SARS-CoV-2 with or without fluorescence tested using RT-PCR. (D) Co-localization of DiO and anti-S antibody (TRITC) signals in pseudo-SARS-CoV-2. Colocalization of anti-S antibody (FITC) and mCherry fluorescent protein signals in pseudo-SARS-CoV-2. Co- 
localization of DiO and mCherry fluorescent protein signals in pseudo-SARS-CoV-2. The insert (right) is a zoomed view of the co-localized dots (Scale bar: $2 \mu \mathrm{m}$ ).

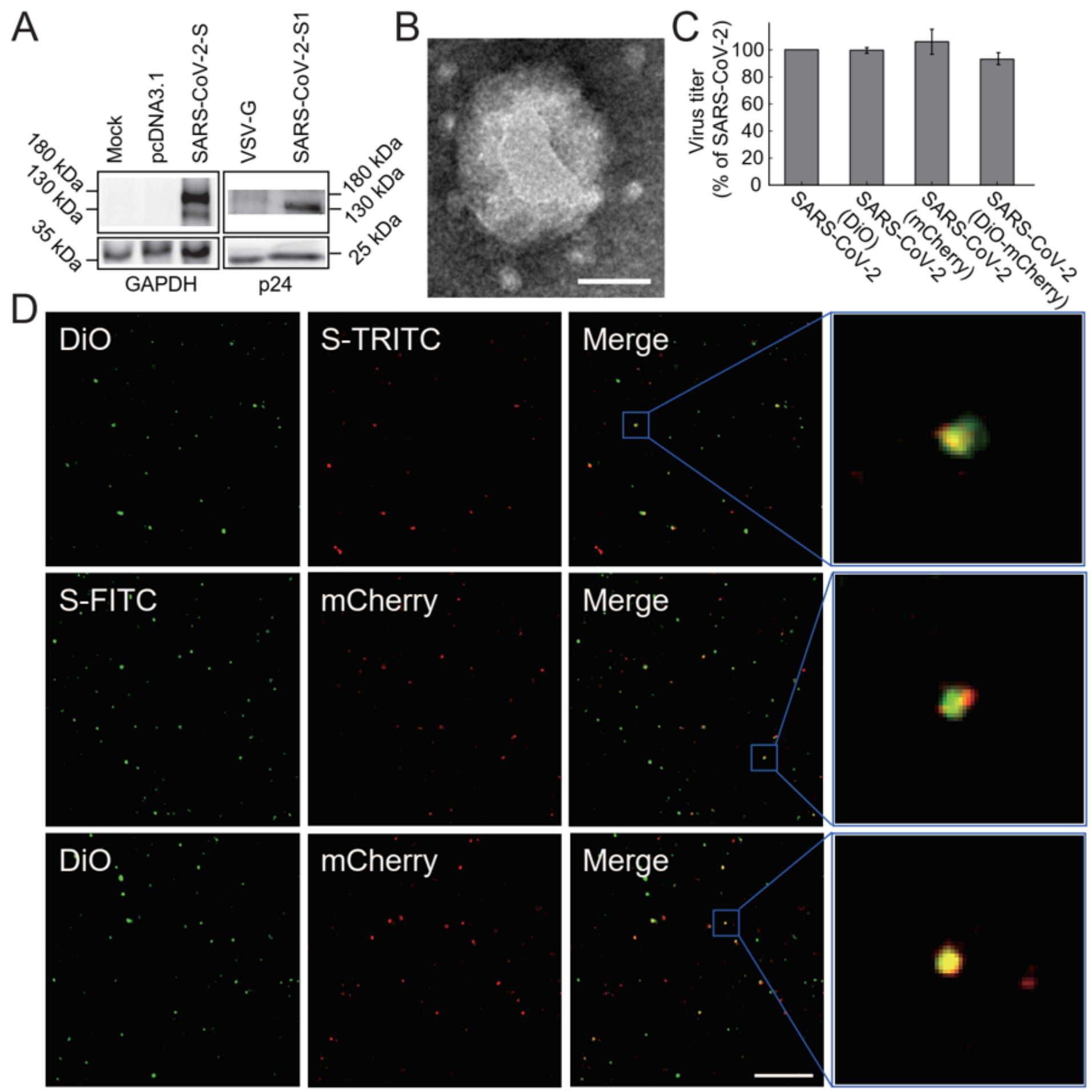

\section{Figure 1}

(A) Western Blot analysis of the SARS-CoV-2 S protein in the cell lysates (left). Western Blot analysis of the SARS-CoV-2 S1 protein in pseudovirions (right). (B) TEM images of the negatively stained pseudoSARS-CoV-2 (Scale bar: $50 \mathrm{~nm}$ ). (C) Titers of pseudo-SARS-CoV-2 with or without fluorescence tested using RT-PCR. (D) Co-localization of DiO and anti-S antibody (TRITC) signals in pseudo-SARS-CoV-2. Co- 
localization of anti-S antibody (FITC) and mCherry fluorescent protein signals in pseudo-SARS-CoV-2. Colocalization of $\mathrm{DiO}$ and mCherry fluorescent protein signals in pseudo-SARS-CoV-2. The insert (right) is a zoomed view of the co-localized dots (Scale bar: $2 \mu \mathrm{m}$ ).
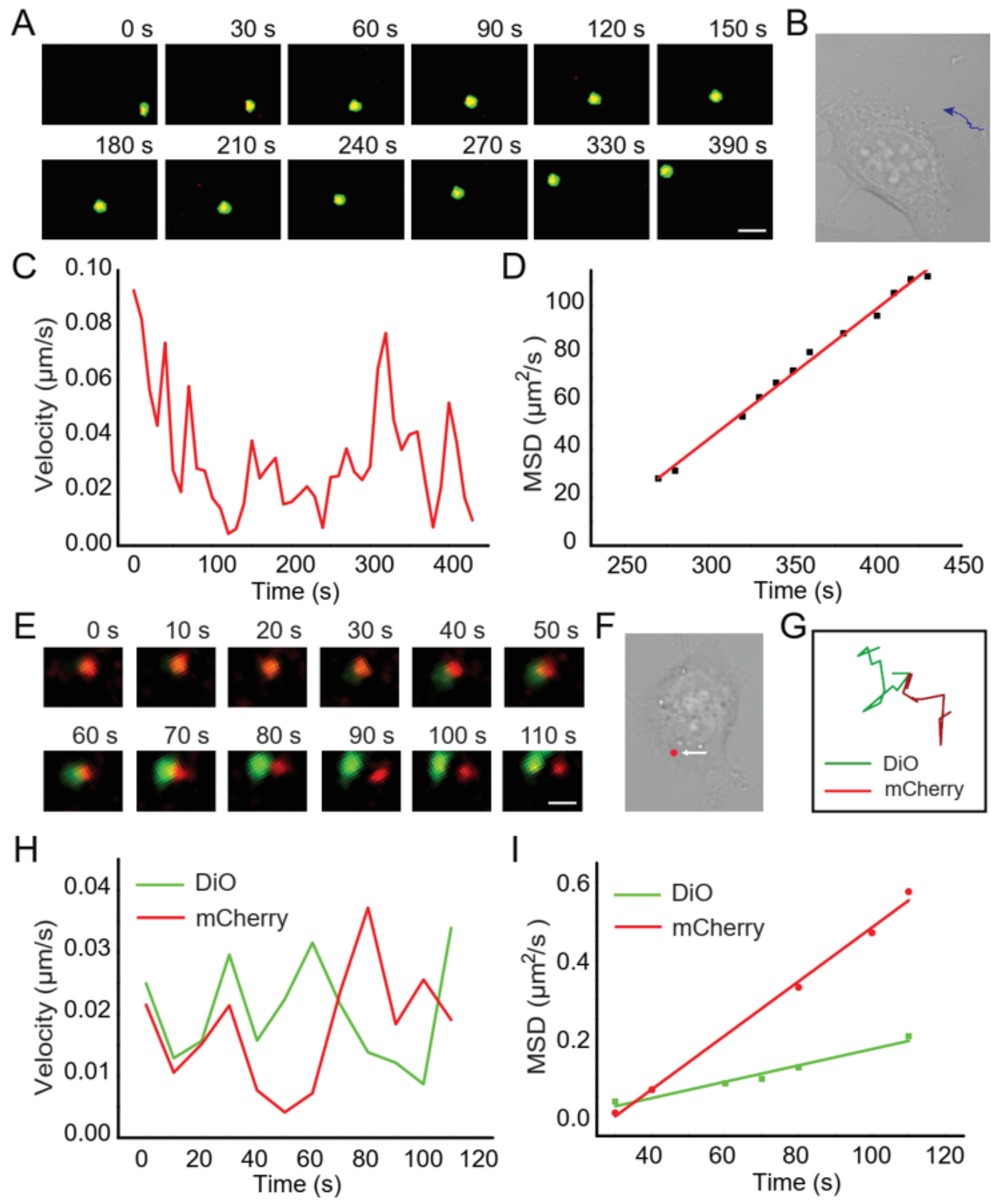

Figure 2

(A) Sequential snapshots of pseudo-SARS-CoV-2 entry into an HNEpC cell (Scale bar: $1 \mu \mathrm{m})$. (B) Differential Interference Contrast (DIC) image of the host cell. The blue line shows the trajectory of the 
virus. (C-D) Analysis of mean velocity (C) and MSD plot (D) of the virus particle shown in (A). (E) Sequential images of the separation of mCherry-Vpr and DiO of pseudo-SARS-CoV-2 in the HNEpC cell (Scale bar: $1 \mu \mathrm{m})$. (F) DIC image of the host cell. The red spot indicates the separation site. (G-I) Trajectories (G), mean velocities $(H)$, and MSD plots (I) of mCherry-Vpr and DiO.
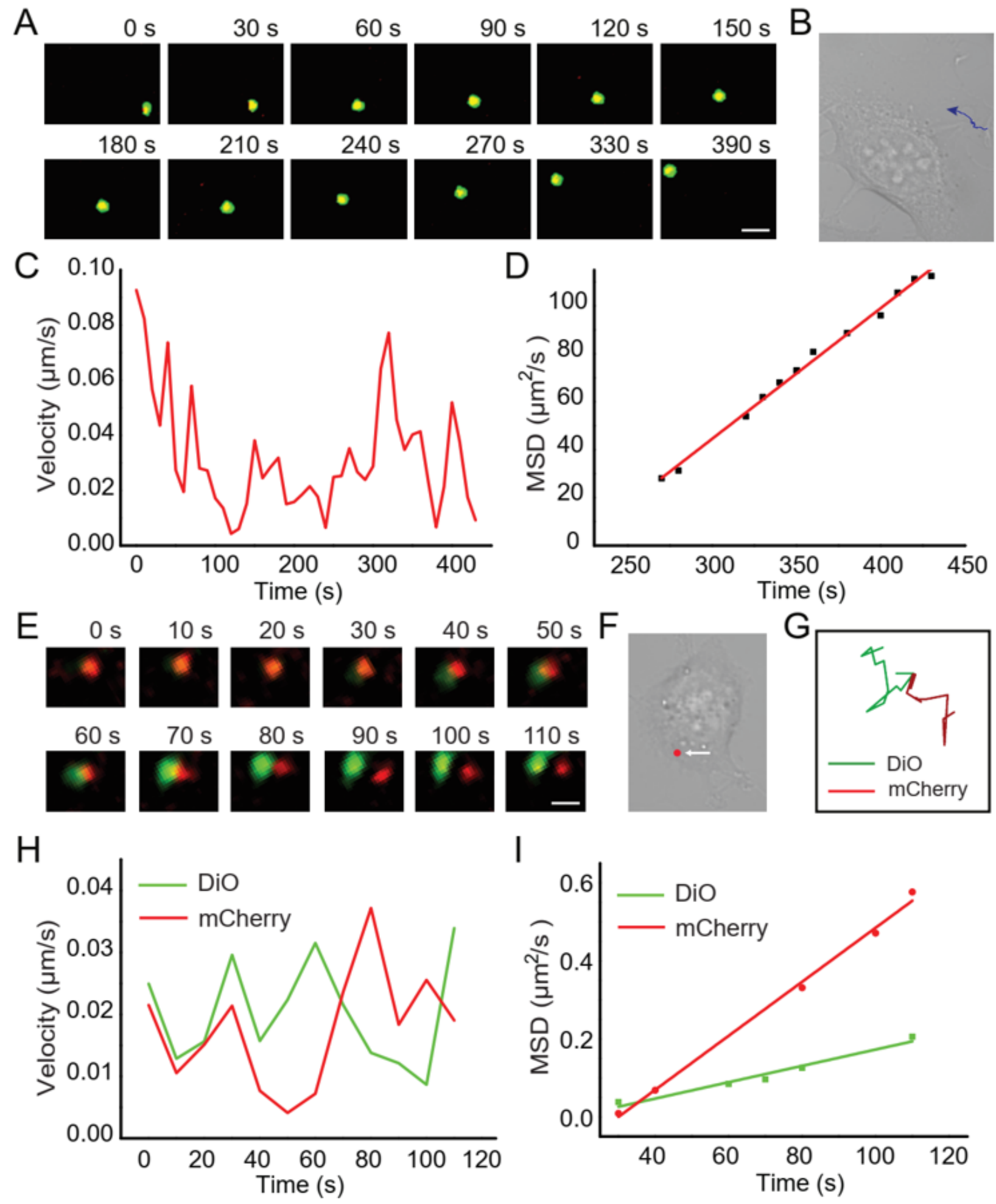

Figure 2 
(A) Sequential snapshots of pseudo-SARS-CoV-2 entry into an HNEpC cell (Scale bar: $1 \mu \mathrm{m})$. (B) Differential Interference Contrast (DIC) image of the host cell. The blue line shows the trajectory of the virus. (C-D) Analysis of mean velocity (C) and MSD plot (D) of the virus particle shown in (A). (E) Sequential images of the separation of mCherry-Vpr and DiO of pseudo-SARS-CoV-2 in the HNEpC cell (Scale bar: $1 \mu \mathrm{m}$ ). (F) DIC image of the host cell. The red spot indicates the separation site. (G-I) Trajectories (G), mean velocities $(H)$, and MSD plots (I) of mCherry-Vpr and DiO.
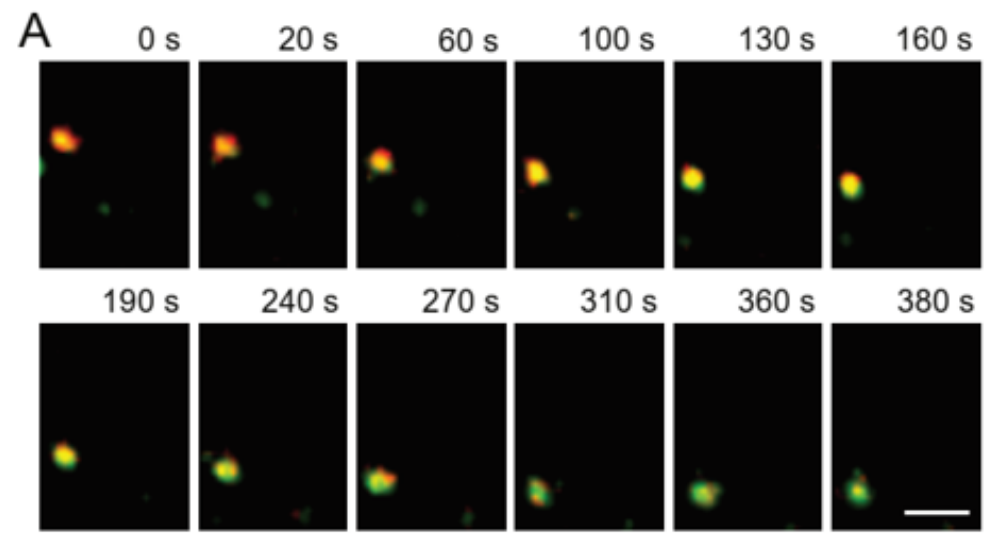

C
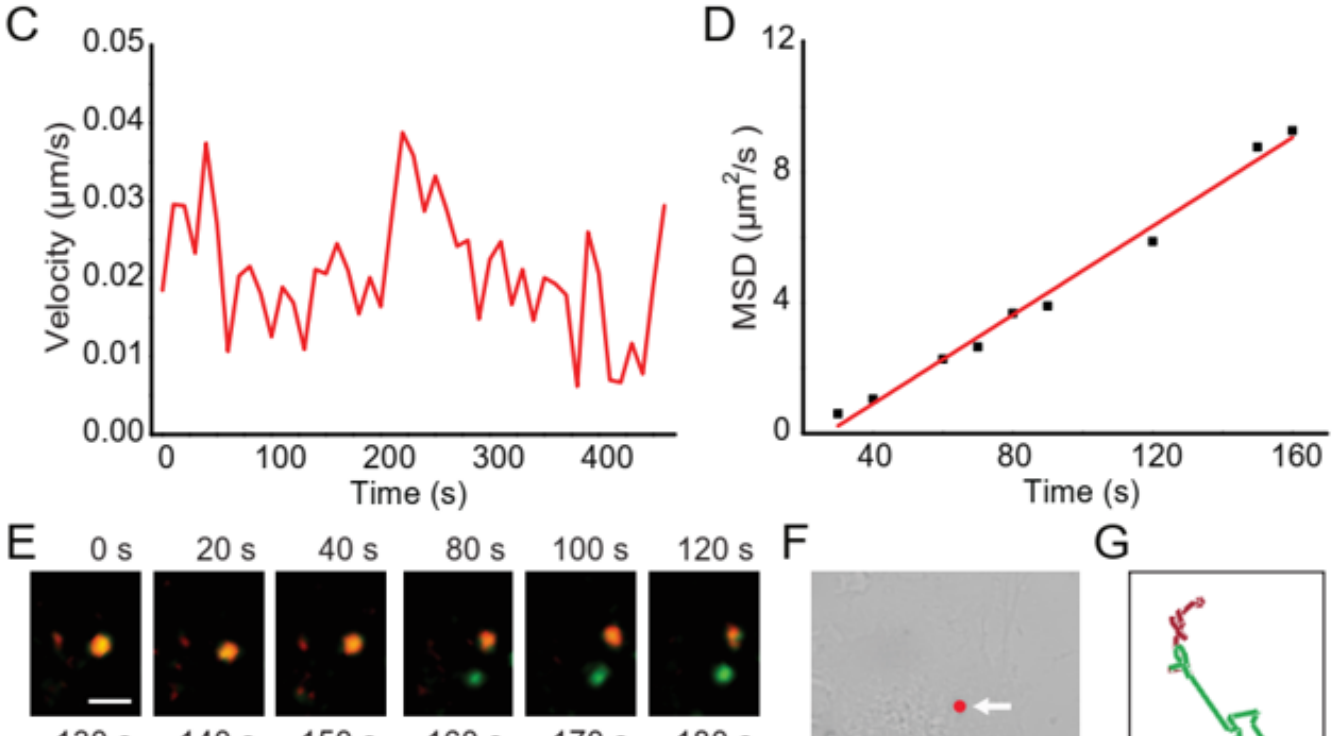

120 s $F$
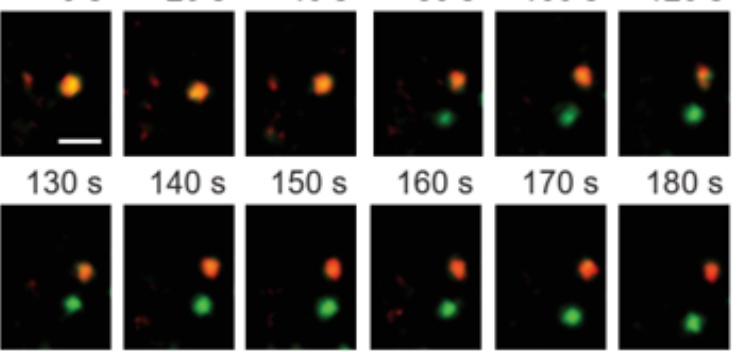

$180 \mathrm{~s}$

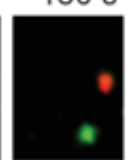

G

$\mathrm{H}$
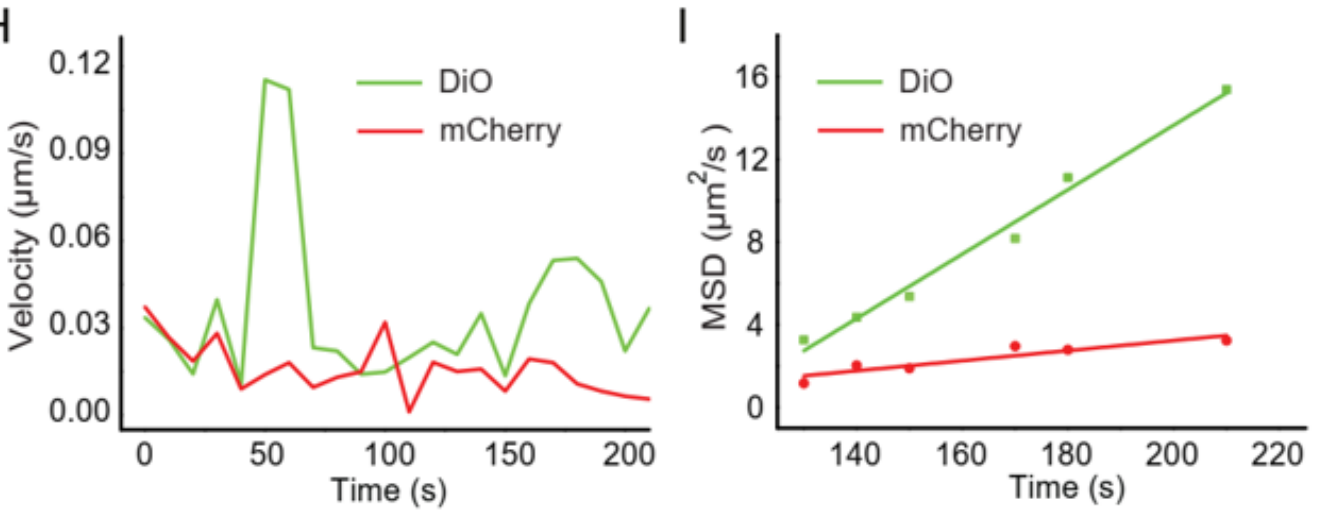

Figure 3 
(A) Sequential snapshots of the entry of pseudo-SARS-CoV-2 into an HPAEpiC cell (Scale bar: $1 \mu \mathrm{m})$. (B) DIC image of the host cell. The blue line shows the trajectory of the virus. (C-D) Analysis of mean velocity (C) and MSD plot (D) of the virus particle shown in (A). (E) Sequential images of the separation of mCherry-Vpr and DiO of pseudo-SARS-CoV-2 in an HPAEpiC cell (Scale bar: $1 \mu \mathrm{m}$ ). (F) DIC image of the host cell. The red spot indicates the separation site. (G-I) Trajectories (G), mean velocities (H), and MSD plots (I) of mCherry-Vpr and DiO.
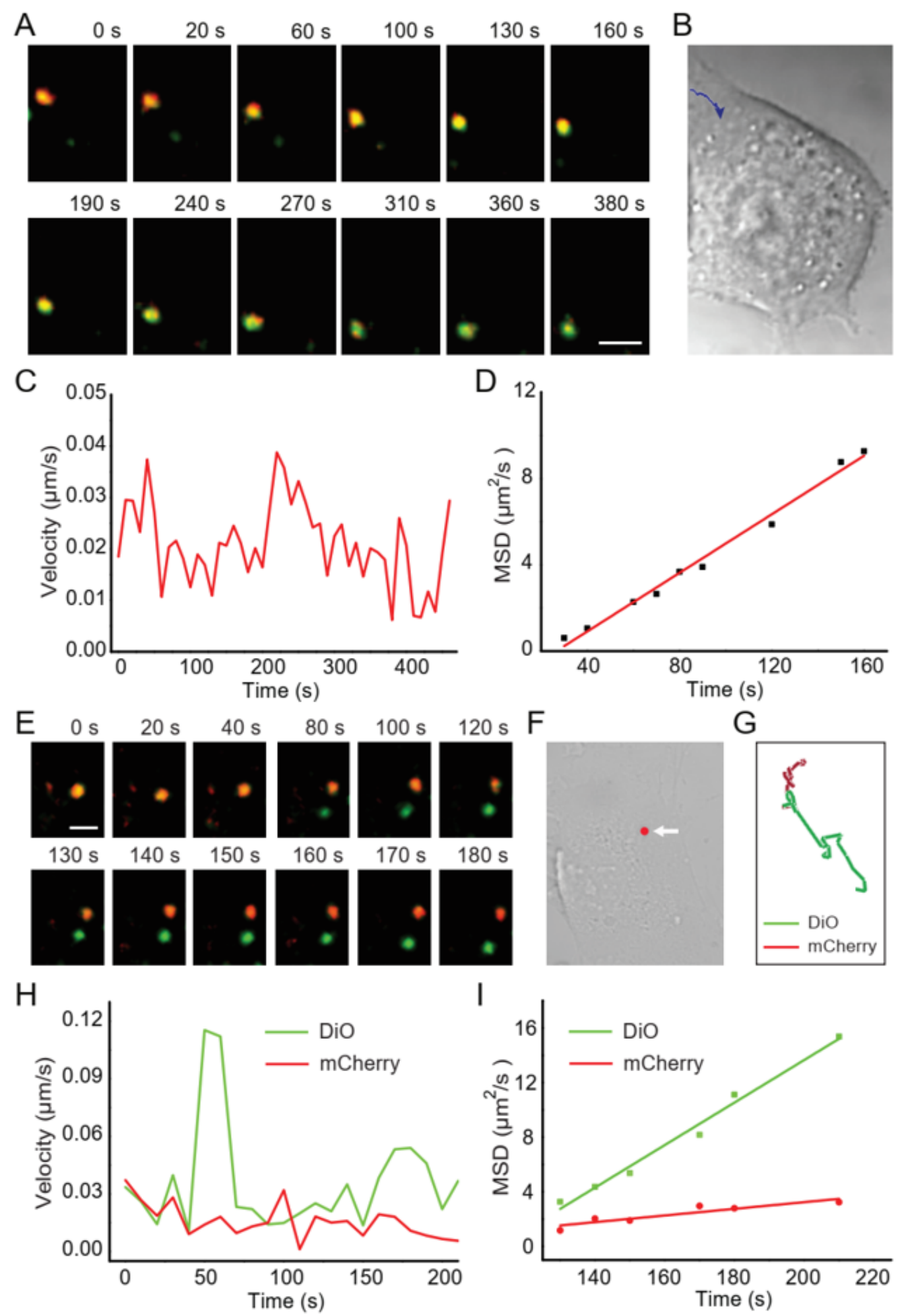

120 s $F$ G
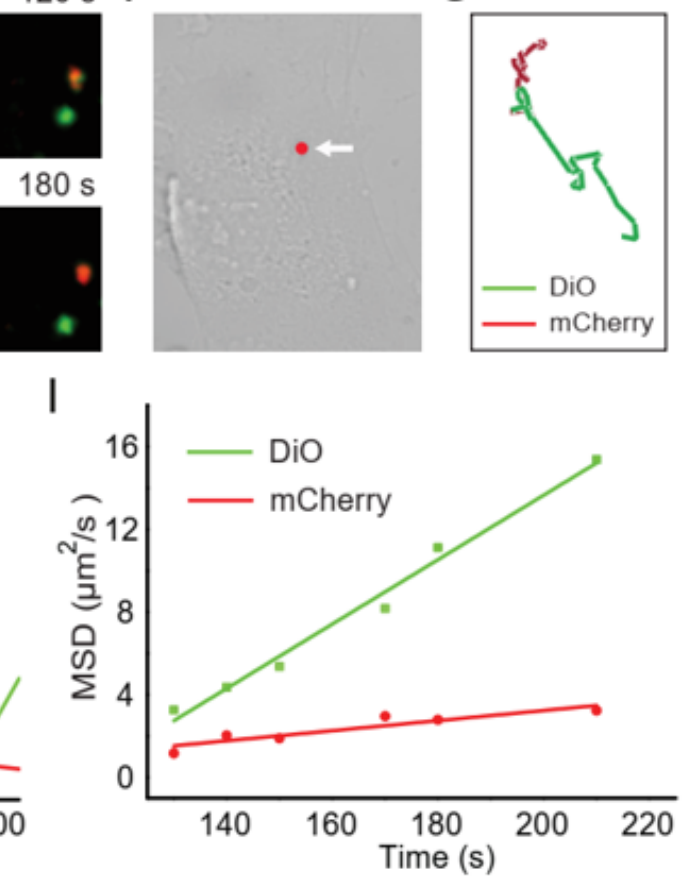

Figure 3 
(A) Sequential snapshots of the entry of pseudo-SARS-CoV-2 into an HPAEpiC cell (Scale bar: $1 \mu \mathrm{m})$. (B) DIC image of the host cell. The blue line shows the trajectory of the virus. (C-D) Analysis of mean velocity (C) and MSD plot (D) of the virus particle shown in (A). (E) Sequential images of the separation of mCherry-Vpr and DiO of pseudo-SARS-CoV-2 in an HPAEpiC cell (Scale bar: $1 \mu \mathrm{m}$ ). (F) DIC image of the host cell. The red spot indicates the separation site. (G-I) Trajectories (G), mean velocities (H), and MSD plots (I) of mCherry-Vpr and DiO.

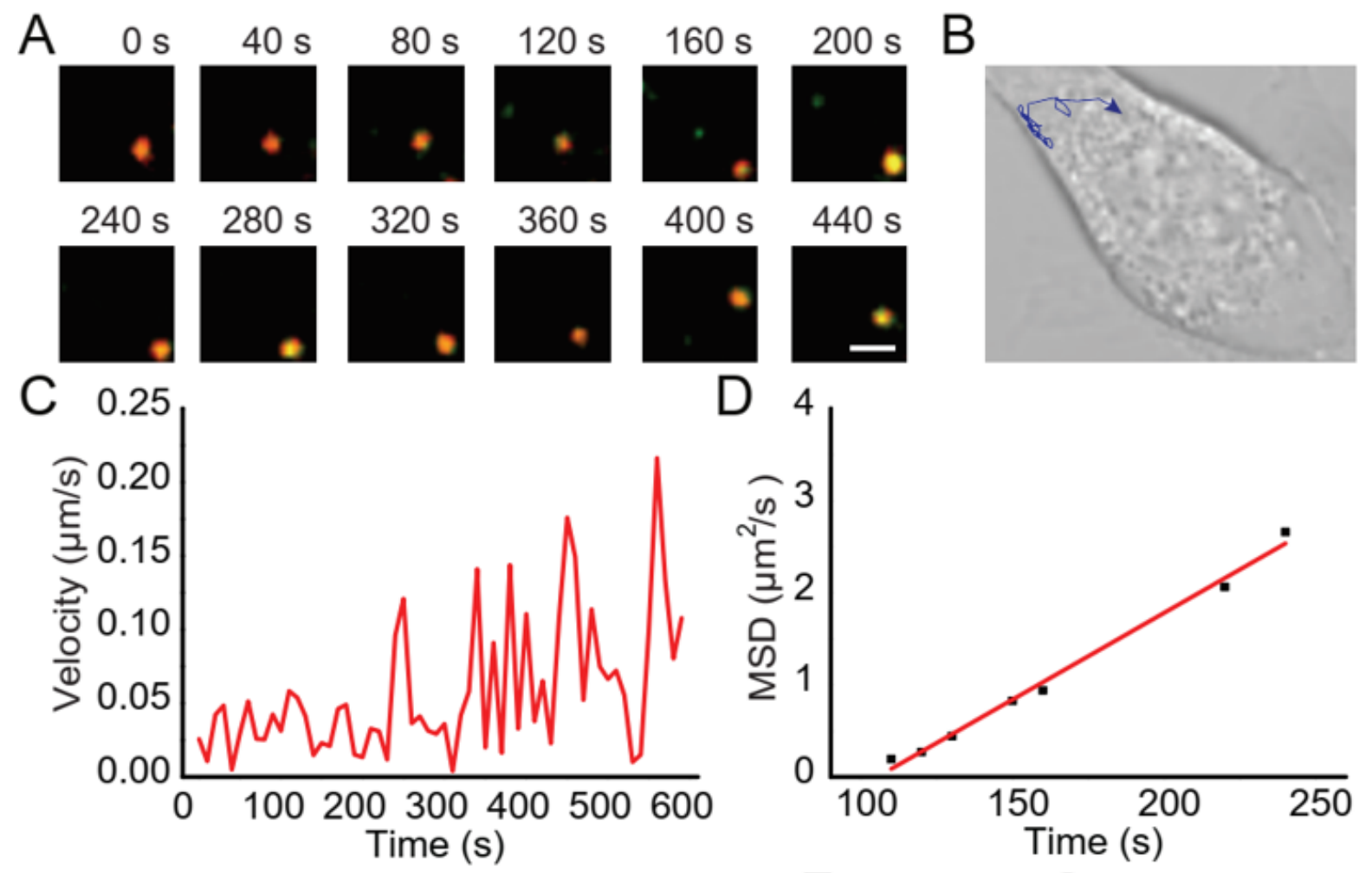

E 0

Time
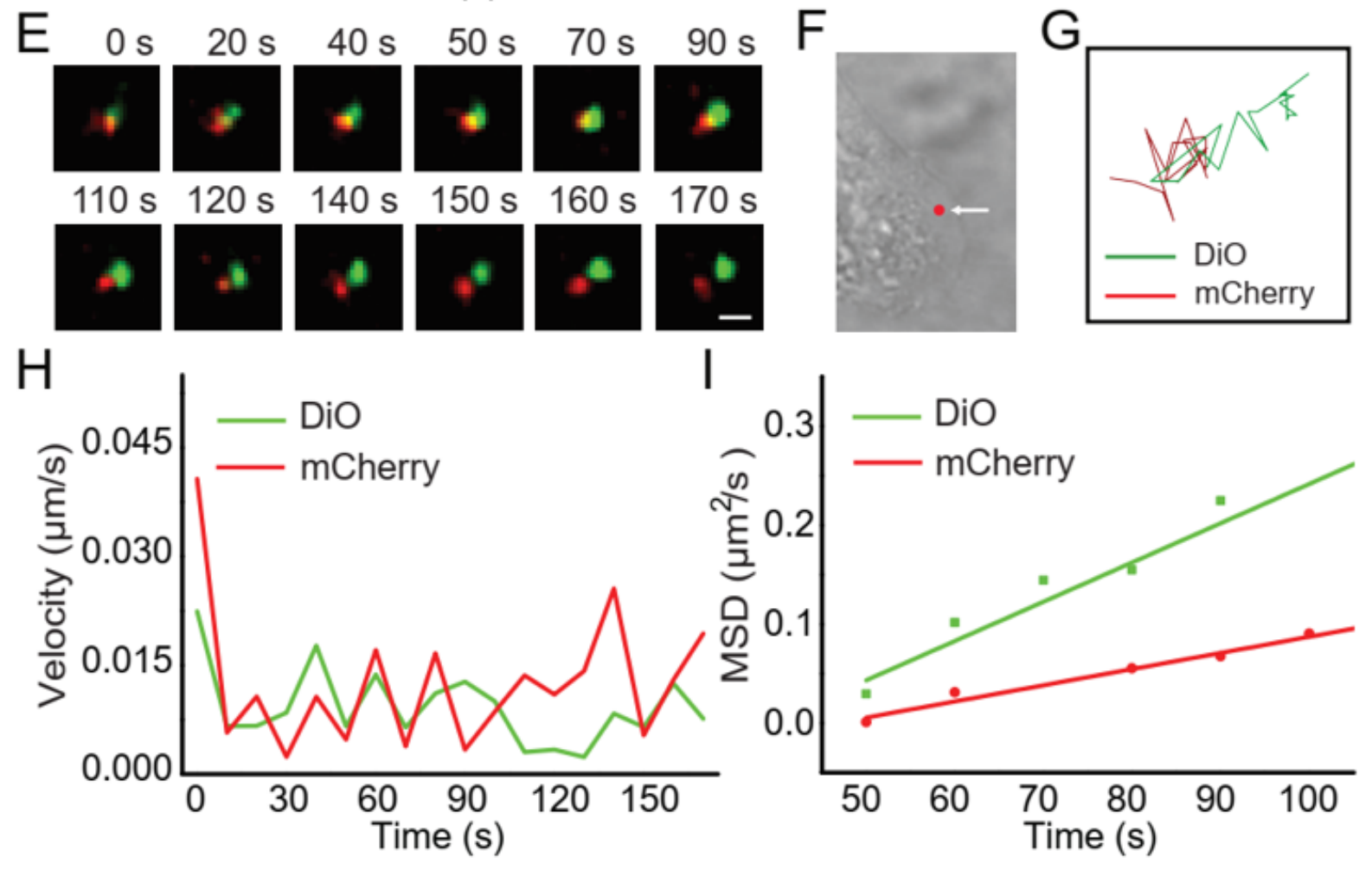

Figure 4 
(A) Sequential snapshots of the entry of pseudo-SARS-CoV-2 into a BEP-2D cell (Scale bar: $1 \mu \mathrm{m})$. (B) DIC image of the host cell. The blue line shows the trajectory of the virus. (C-D) Analysis of mean velocity (C) and MSD plot (D) of the virus particle shown in (A). (E) Sequential images of the separation of mCherryVpr and DiO of pseudo-SARS-CoV-2 in a BEP-2D cell (Scale bar: $1 \mu \mathrm{m}$ ). (F) DIC image of the host cell. The red spot indicates the separation site. (G-I) Trajectories (G), mean velocities (H), and MSD plots (I) of mCherry-Vpr and DiO.

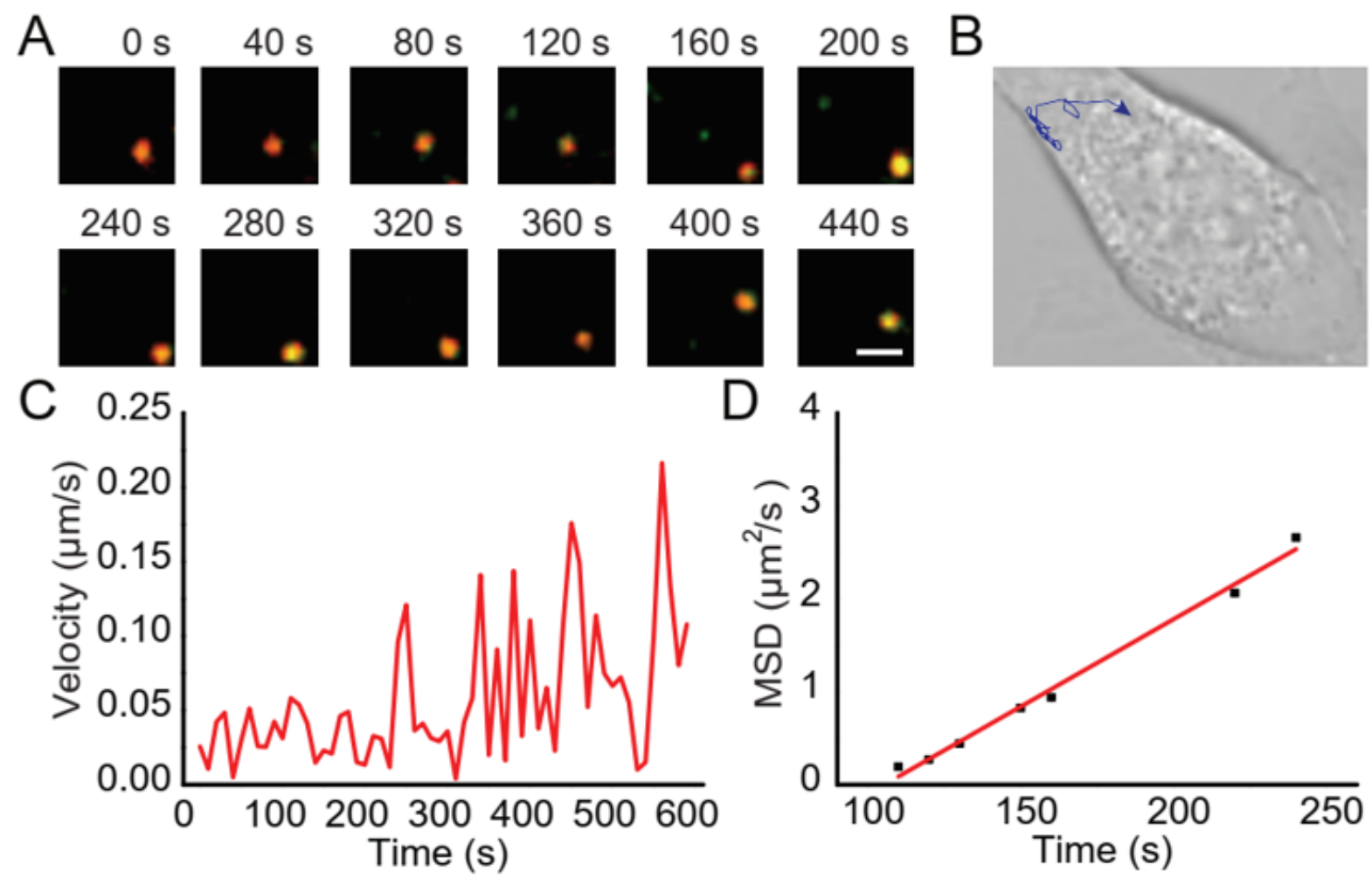

E 0

Time (s)
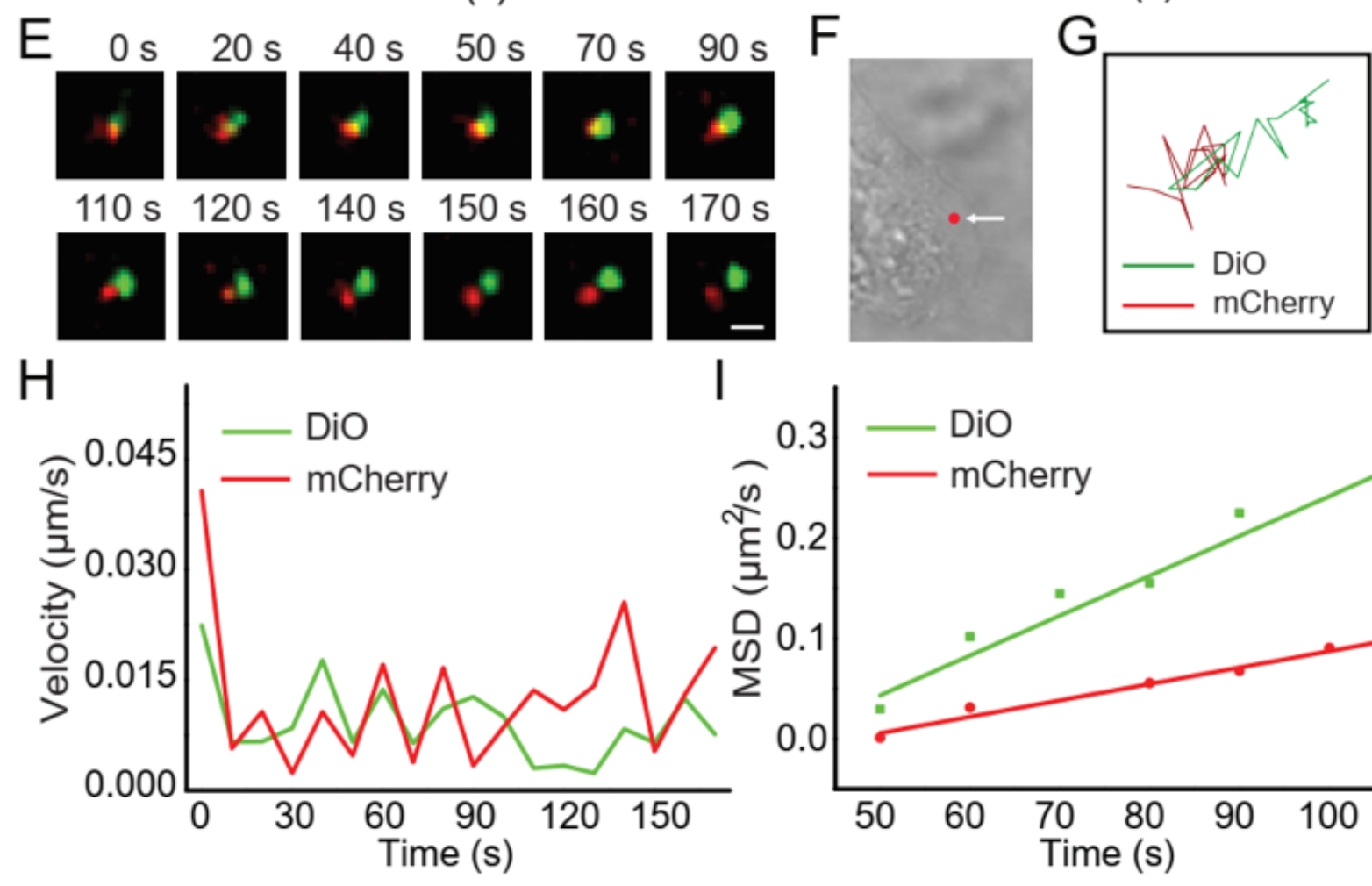

$\mathrm{H}$

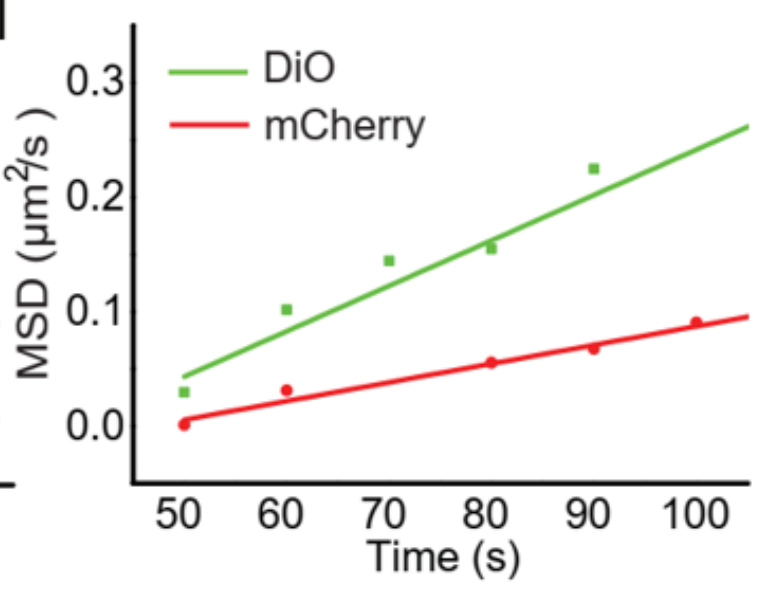

Figure 4 
(A) Sequential snapshots of the entry of pseudo-SARS-CoV-2 into a BEP-2D cell (Scale bar: $1 \mu \mathrm{m}$ ). (B) DIC image of the host cell. The blue line shows the trajectory of the virus. (C-D) Analysis of mean velocity (C) and MSD plot (D) of the virus particle shown in (A). (E) Sequential images of the separation of mCherryVpr and DiO of pseudo-SARS-CoV-2 in a BEP-2D cell (Scale bar: $1 \mu \mathrm{m}$ ). (F) DIC image of the host cell. The red spot indicates the separation site. (G-I) Trajectories (G), mean velocities (H), and MSD plots (I) of mCherry-Vpr and DiO.
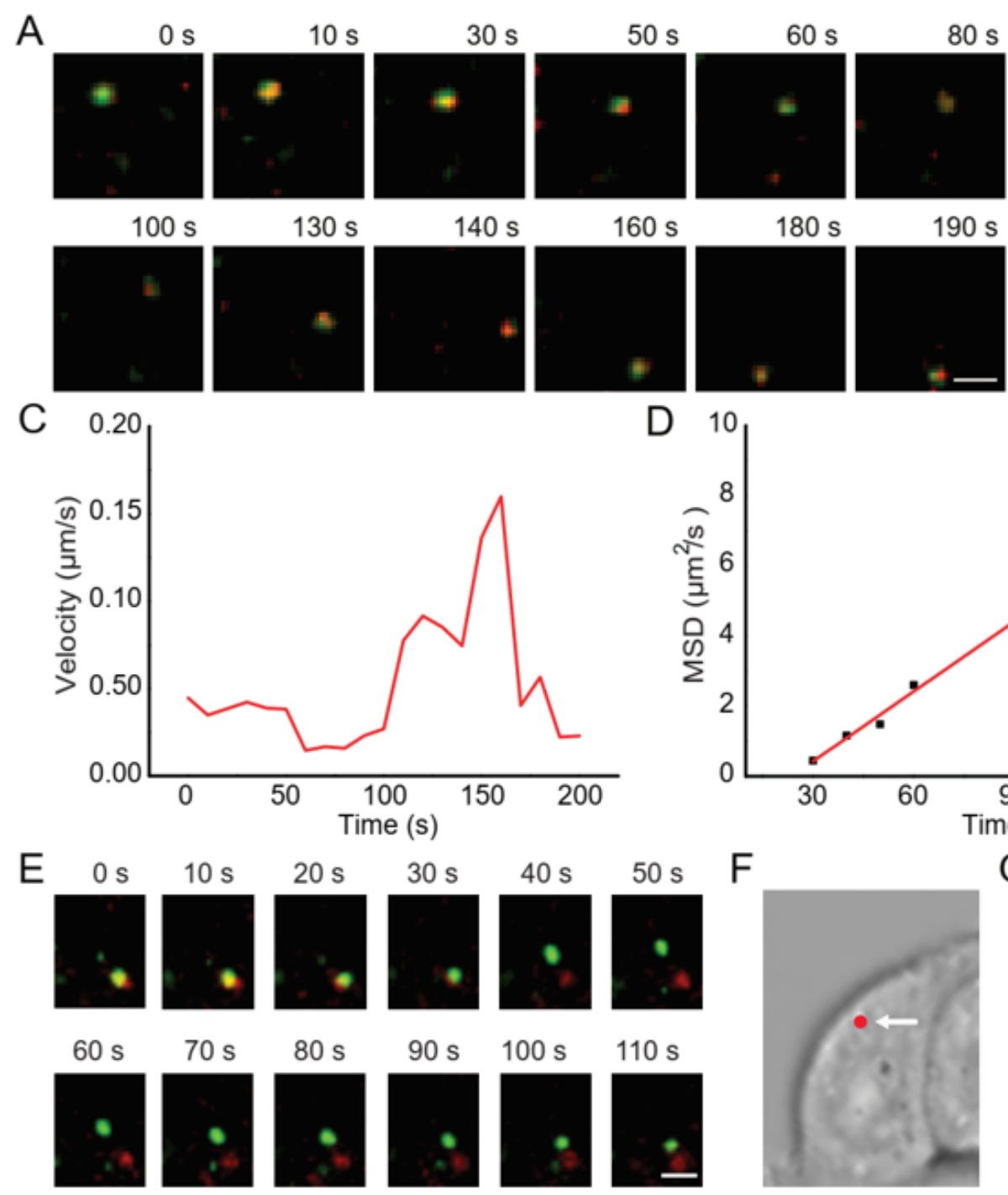

B

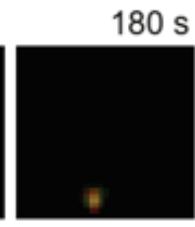

$190 \mathrm{~s}$
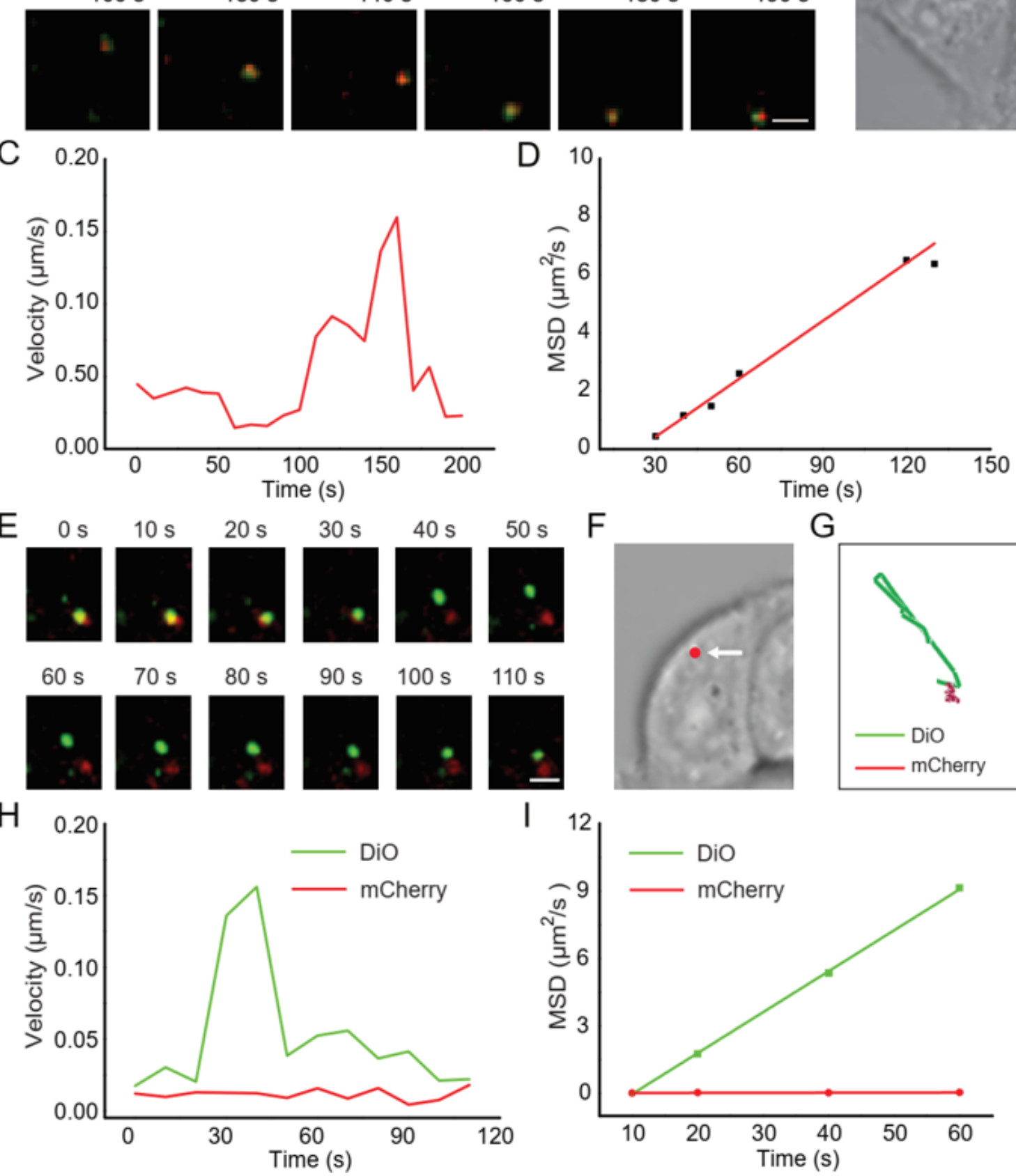

Figure 5 
(A) Sequential snapshots of the entry of pseudo-SARS-CoV-2 into a HOEC cell (Scale bar: $1 \mu \mathrm{m})$. (B) DIC image of the host cell. The blue line shows the trajectory of the virus. (C-D) Analysis of mean velocity (C) and MSD plot (D) of the virus particle shown in (A). (E) Sequential images of the separation of mCherryVpr and DiO of pseudo-SARS-CoV-2 in a HOEC cell (Scale bar: $1 \mu \mathrm{m}$ ). (F) DIC image of the host cell. The red spot indicates the separation site. (G-I) Trajectories (G), mean velocities (H), and MSD plots (I) of mCherry-Vpr and DiO.
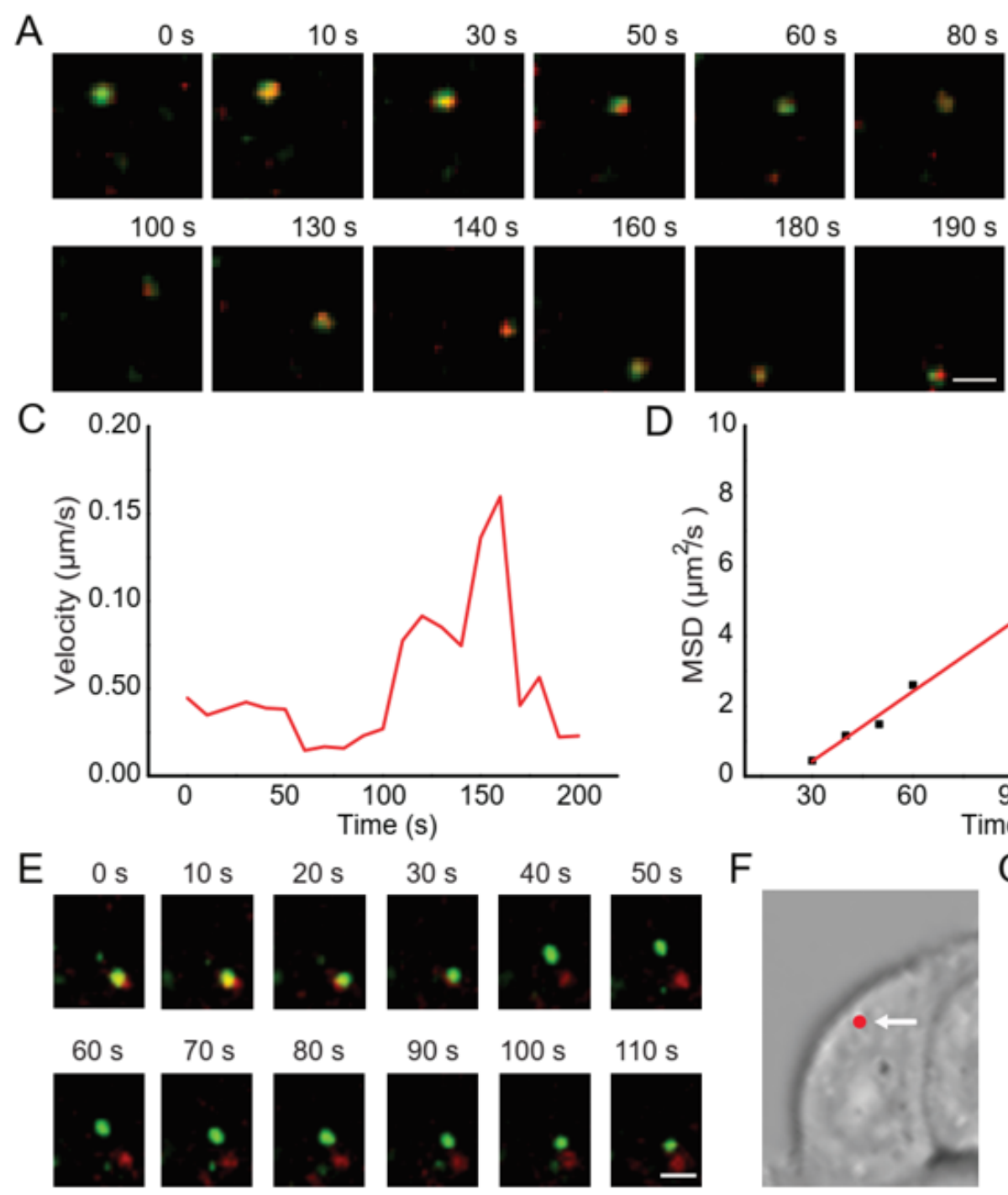

B

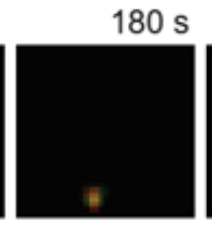

$190 \mathrm{~s}$
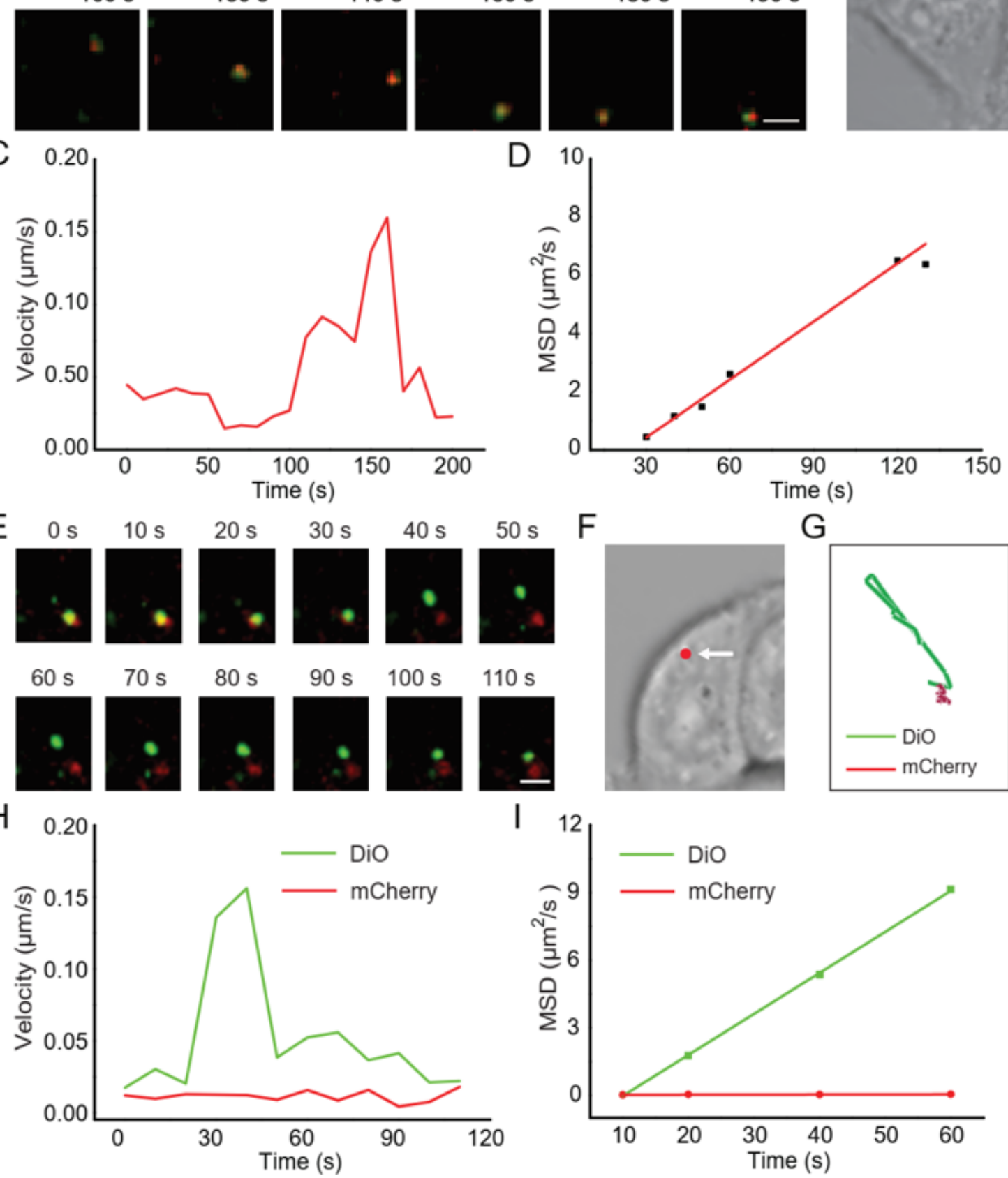

Figure 5 
(A) Sequential snapshots of the entry of pseudo-SARS-CoV-2 into a HOEC cell (Scale bar: $1 \mu \mathrm{m}$ ). (B) DIC image of the host cell. The blue line shows the trajectory of the virus. (C-D) Analysis of mean velocity (C) and MSD plot (D) of the virus particle shown in (A). (E) Sequential images of the separation of mCherryVpr and DiO of pseudo-SARS-CoV-2 in a HOEC cell (Scale bar: $1 \mu \mathrm{m}$ ). (F) DIC image of the host cell. The red spot indicates the separation site. (G-I) Trajectories (G), mean velocities (H), and MSD plots (I) of mCherry-Vpr and DiO.
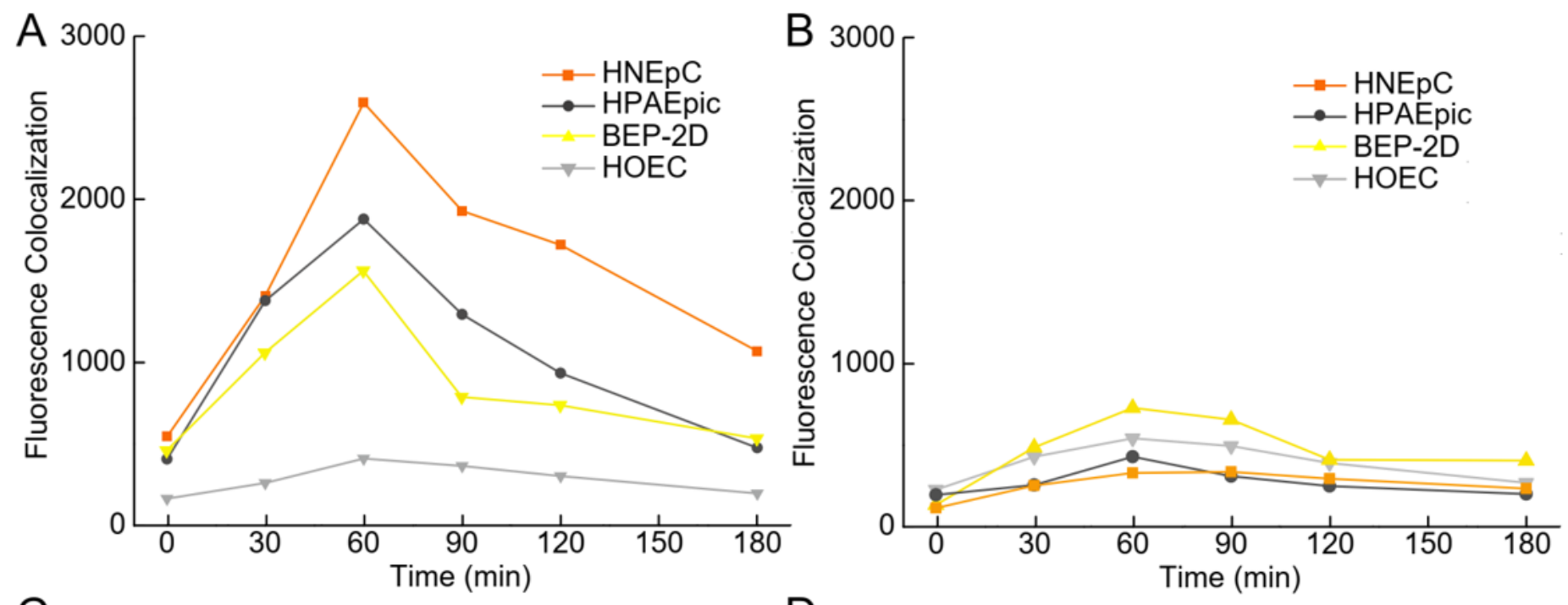

C

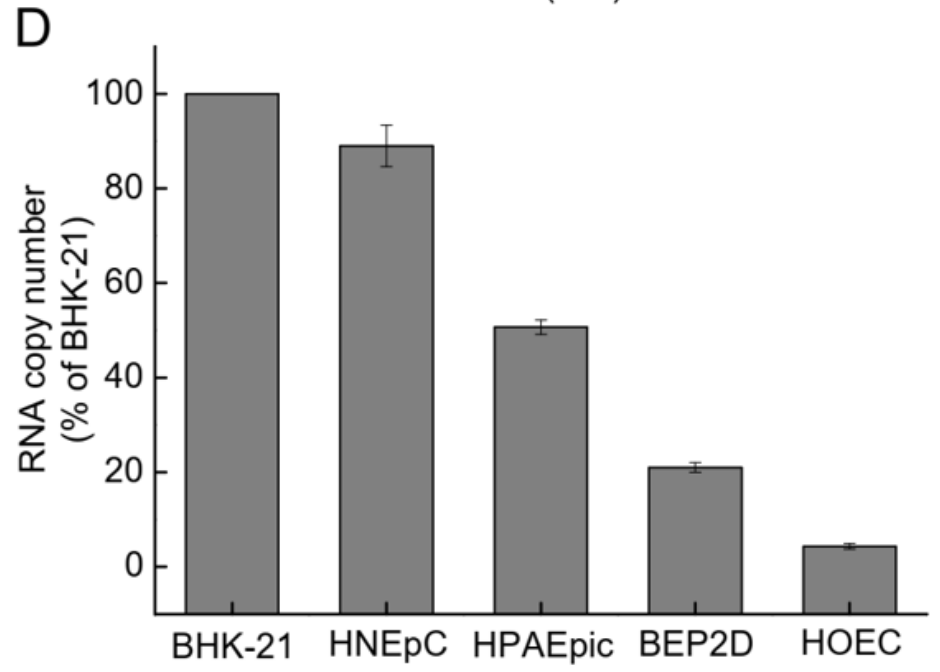

Figure 6

(A) Statistical analysis of the dual-fluorescent pseudo-SARS-CoV-2 in respiratory epithelial cells, which were fixed for 0-180 min post-infection. (B) Statistical analysis of the dual-fluorescent VSV-G pseudovirions in respiratory epithelial cells, which were fixed for 0-180 min post-infection. (C) Western blot analysis of ACE2 receptor in the BHK-21 cells as well as different respiratory epithelial cells. (D) RTPCR analysis of the intracellular viral RNA copies, representing SARS-CoV-2 entry. 

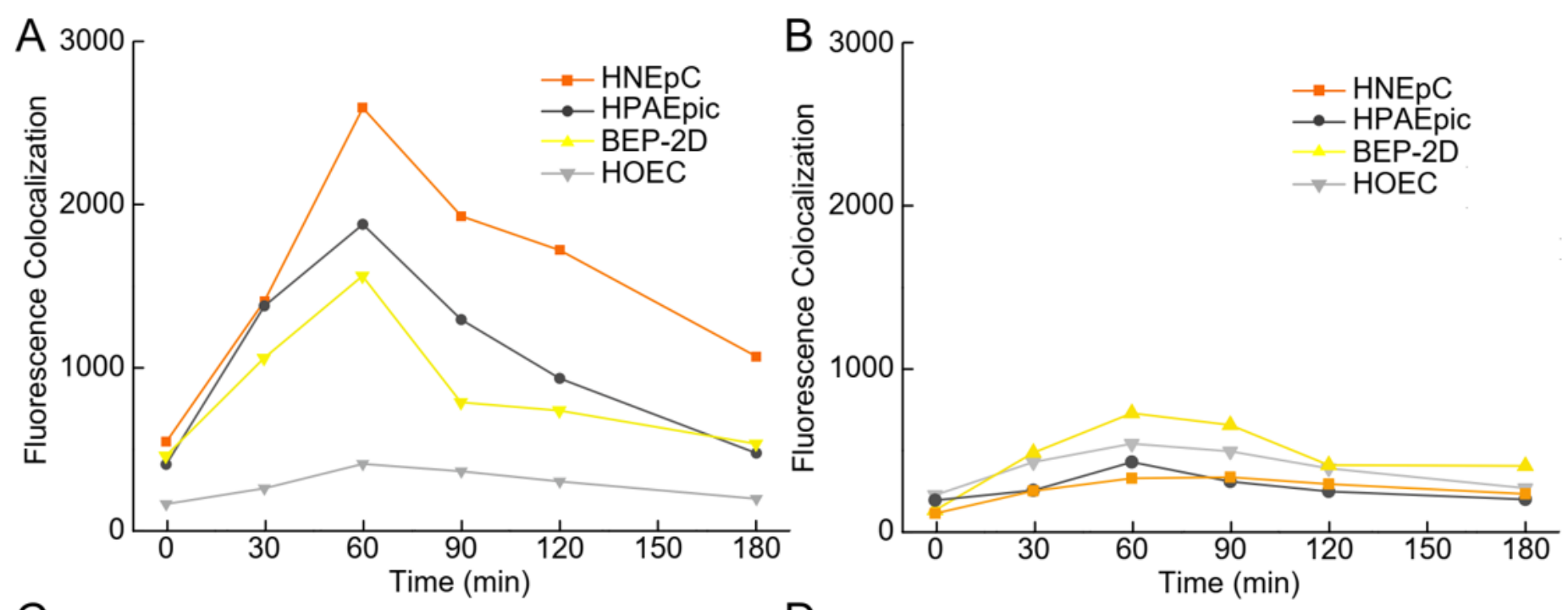

C

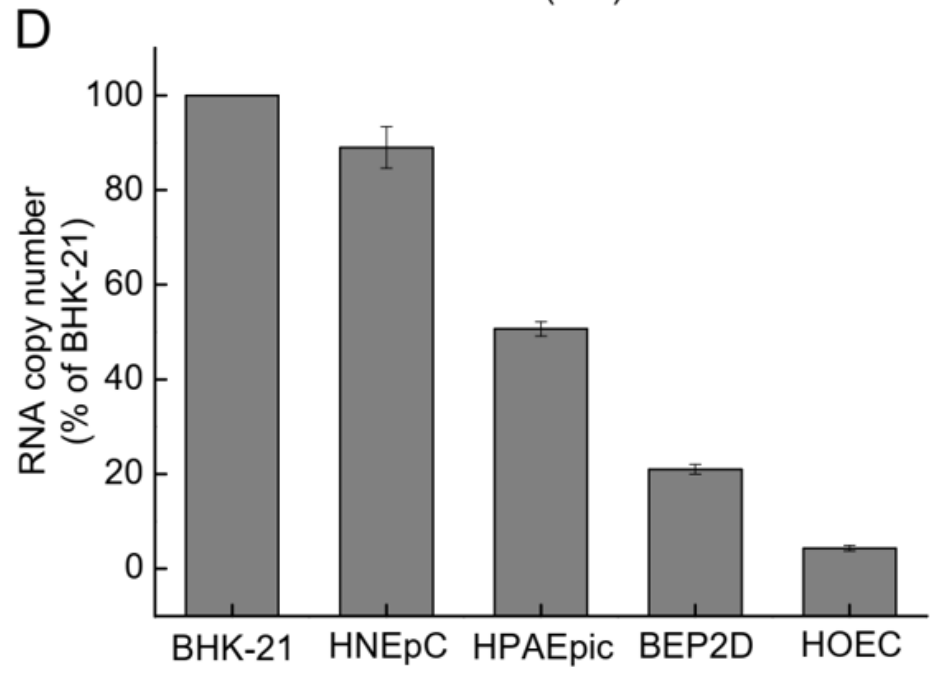

\section{Figure 6}

(A) Statistical analysis of the dual-fluorescent pseudo-SARS-CoV-2 in respiratory epithelial cells, which were fixed for 0-180 min post-infection. (B) Statistical analysis of the dual-fluorescent VSV-G pseudovirions in respiratory epithelial cells, which were fixed for 0-180 min post-infection. (C) Western blot analysis of ACE2 receptor in the BHK-21 cells as well as different respiratory epithelial cells. (D) RTPCR analysis of the intracellular viral RNA copies, representing SARS-CoV-2 entry.

\section{Supplementary Files}

This is a list of supplementary files associated with this preprint. Click to download.

- MovieS1HNEPC1.mov

- MovieS1HNEPC1.mov

- MovieS2HNEPC2.mov 
- MovieS2HNEPC2.mov

- MovieS3HPAEpiC1.mov

- MovieS3HPAEpiC1.mov

- MovieS4HPAEpiC2.mov

- MovieS4HPAEpiC2.mov

- MovieS5BEP2D1.mov

- MovieS5BEP2D1.mov

- MovieS6BEP2D2.mov

- MovieS6BEP2D2.mov

- MovieS7HOEC1.mov

- MovieS7HOEC1.mov

- MovieS8HOEC2.mov

- MovieS8HOEC2.mov

- MovieS9HNEPCVSVG1.mov

- MovieS9HNEPCVSVG1.mov

- MovieS10HNEPCVSVG2.mov

- MovieS10HNEPCVSVG2.mov

- MovieS11HPAEpicVSVG1.mov

- MovieS11HPAEpicVSVG1.mov

- MovieS12HPAEpicVSVG2.mov

- MovieS12HPAEpicVSVG2.mov

- MovieS13BEP2DVSVG1.mov

- MovieS13BEP2DVSVG1.mov

- MovieS14BEP2DVSVG2.mov

- MovieS14BEP2DVSVG2.mov

- MovieS15HOECVSVG1.mov

- MovieS15HOECVSVG1.mov

- MovieS16HOECVSVG2.mov

- MovieS16HOECVSVG2.mov

- SARSCoV2SI.docx

- SARSCoV2SI.docx 